Historic, Archive Document

Do not assume content reflects current scientific knowledge, policies, or practices. 


\section{冬tillman's}

\section{PRICE LIST FOR}

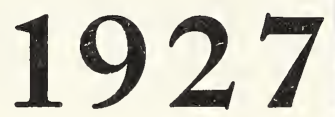

GOING OUT OF BUSINESS

Great Reductions in Prices

Rich Bargains for Customers

See Following Page

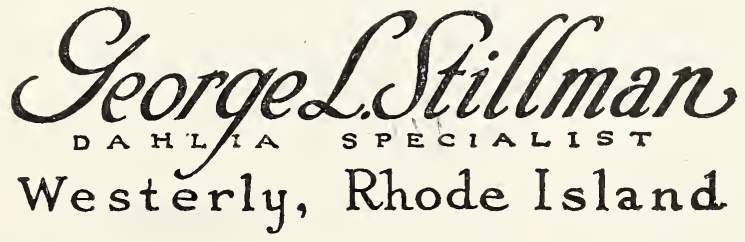




\section{An Explanation}

QLL health nearly a year, a critical operation in the hospital, and my age (70 years) compel me to retire from all business responsibility.

My entire original collection of WORLD Renowned Dahlias must be sold at some price.

Prices are given herein for single divisions, and dozens of one kind. Write for prices of larger lots or make offers for the entire stock of any variety.

\section{CASH WITH ORDER}

I have taken nearly twice as many orders from my field this fall for spring delivery as ever before, and indications are that the dahlia business this year will surpass all records.

Address all orders and communications to Geo. L. Stillman, Dahlia Specialist, Westerly, Rhode Island. IMPORTANT.- It is essential that you use the initial "L." as there are several Geo. Stillmans in town, but no other "Stillman" growing Dahlias. 


\title{
ABBREVIATIONS OF CLASSES
}

\author{
Cactus (C) \\ Show ( $\mathrm{SH})$ \\ Decorative (D) \\ Single (S) \\ Hybrid Cactus (HC) \\ Paeony (P) \\ Pompom (PP) \\ Cactus Paeony (CP) \\ No Classifications (-)
}

If ordered in clumps before divided, double the price of one bulb.

No. of

NAME - CLASS - DESCRIPTION

$\underset{\text { perice Bulb }}{\text { Per }}$

Price
er Dozen

Total No. Divisions

\section{INDIAN FAMILY}

107 Blackhawk (P) Deep velvet maroon ......... $\$ 1.00$

$\$ 5.00$

214

Canonchet (P) Salmon, suffused gold ........

1.00

5.00

16

246 Canonicus

(P) Dark cerise $\ldots \ldots \ldots \ldots \ldots \ldots$

1.00

5.00

1.00

5.00

492

P) Maroon tipped white .........

1.00

5.00

90

Minnewawa $(P)$ Cerise red, tipped white .....

1.00

5.00

898

555

Misquamicut

(P) Yellow, tinged pink ........

1.00

5.00

66

1.00

5.00

1110

79
432

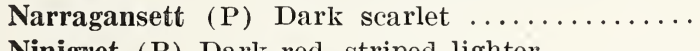

1.00

Nokomis (P) Yellow and white with red .......

1.00

5.00

1.00

5.00

158

138

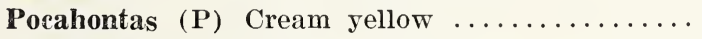

1.00

5.00

864

24

Powhatan (P) Crushed strawberry ..........

1.00

5.00

103

Queen Esther (P) "DuBarry" red ...........

1.00

5.00

48

82

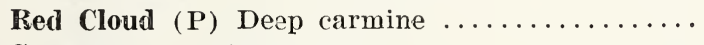

1.00

5.00

206

490

Samoset (P) Light yellow $\ldots \ldots \ldots \ldots \ldots \ldots \ldots \ldots$

1.00

5.00

164

169

Uncas (P) Pure white $\ldots \ldots \ldots \ldots \ldots \ldots \ldots \ldots$

1.00

5.00

980

74 Wadawannock

(P) Light coral

5.00

338

148

\section{THE LOMA FAMILY}

510

167

12

385

143

254

368

32

326

42

24

774

Edna Rollins (P) Rose streaked yellow .........

1.00

1.00

1.00

1.00

1.00

1.00

1.00

1.00

1.00

1.00

1.00

1.00

1.00

1.00

1.00

1.00

1.00

1.00
5.00

1020

5.00

5.00

334

5.00

5.00

5.00

5.00

5.00

5.00

5.00

5.00

5.00

5.00

5.00

5.00

5.00

5.00

5.00
24

770

286

508

736

64

652

84 


\section{George L. Stiliman's Dahlia PRICE LIST}

No. of
Clumps NAME - CLASS - DESCRIPTION

\section{THE FIGHTING TEN}

\begin{tabular}{|c|c|c|c|}
\hline 3.3 & Gen. Bell (P) Pure white & 2.00 & 10.00 \\
\hline 96 & Gen. Edwarts (P) Salmon pink & 2.00 & 10.00 \\
\hline 9 & Gen. Cadorna (P) Deep yellow ............ & 2.00 & 10.00 \\
\hline 95 & Gen. Haig (D) Peach, pink, cloucied yellow ... & 2.00 & 10.00 \\
\hline 111 & Gen. Jofire (D) Light canary yellow ........ & 2.00 & 10.00 \\
\hline $4 !$ & 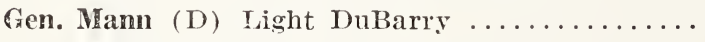 & 2.00 & 10.00 \\
\hline 28 & Gen. Maurice $(P)$ Bright scarlet $\ldots \ldots \ldots \ldots$ & 2.00 & 10.00 \\
\hline 1 & Gen. Pershing (C) Light carmine $\ldots \ldots \ldots \ldots \ldots$ & 2.00 & 10.00 \\
\hline $4 \pi$ & 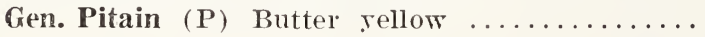 & 2.00 & 10.00 \\
\hline
\end{tabular}

\section{THE TEN SEA LIONS}

1.) Aquitania (C) Salmon pink ............ 1.50

$\begin{array}{rr}8.00 & 30 \\ 8.00 & 10 \\ 8.00 & 470 \\ 8.00 & 136 \\ 8.00 & 172 \\ 8.00 & 188 \\ 8.00 & 22\end{array}$

\section{THE NEW YORK GROUP}

151 Biltmore (D) Carmine tipped white $\ldots \ldots \ldots \ldots \quad 2.00$

10.00

382

26 Eelmont (CP) Deep magenta ............ $2.00 \quad 10.00$

\section{THE GREAT LAKE GROUP}

47 Lake Superior (HC) Orange salmon ......... 3.00

91 Lake Erie (D) Deep lavender ........... 3.00

15.00

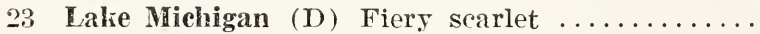

$3.00 \quad 15.00$

\section{THE OCEAN GROUP}

S6 Arctic Ocean (P) Peach pink ........... 5.00

31 Indian Ocean (D.) Red, clouded rellow ...... 5.00 


\section{George L. Stillman's Dahlia PRICE LIS'T}

No. of NAME - CLASS - DESCRIPTION

$\begin{array}{ccc}\text { Price } & \text { Price } & \text { Total No. } \\ \text { per Bulb } & \text { per bozen } & \text { Divisions }\end{array}$

\section{THE FAMOUS RHODE ISLAND GROUP}

127 William Ellery (D) Cerise carmine ........ 15.00

$\begin{array}{rr}75.00 & 25 t \\ 75.00 & 248 \\ 75.00 & 70 \\ 75.00 & 396 \\ 75.00 & 112\end{array}$

1:4 Stephen Hopkins (D) Reddish salmon ........ 15.00

35 Gilbert Stuart (P) Deep lilac ............. 15.00 70

19S Commodore Perry (D) Canary yellow ........ 15.00

56 Nathaniel Greene (D) Yellow veined salmon .. 15.00

112

\section{NEW PAEONY FLOWERED}

226 Adele - Canary yellow ............... .25

251 Ad. Dewey - Strawberry, tipped yellow ...... .50

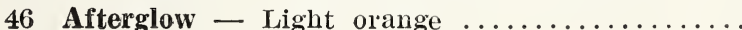

21 Alleghany Mts. - Carmine, tipped white ..... 25.00

24 Alpena - Red, slightly tipped yellow .........

18 Augusta - Bright red ................ 2.00

104 Aunt Basha - Deep maroon ..............

$$
\text { Autumn - Light carmine } \ldots \ldots \ldots \ldots \ldots \ldots
$$

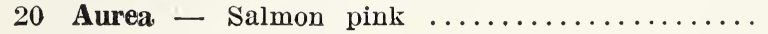

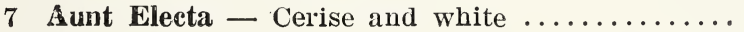

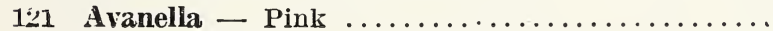

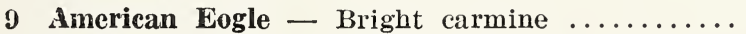

$$
\text { Bellflower - Pink, clouded white } \ldots \ldots \ldots \ldots \ldots
$$

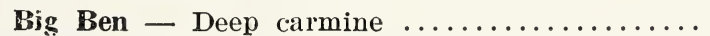

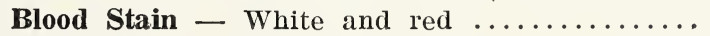

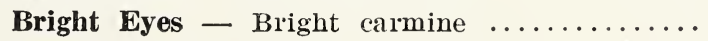

Cardinal Mercier - Pink, streaked yellow ......

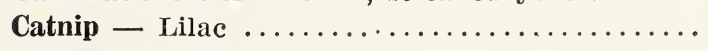

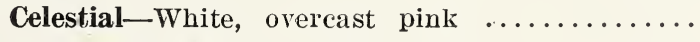

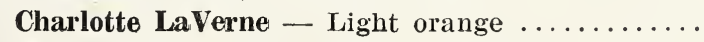

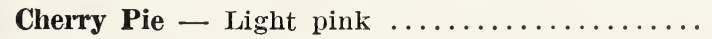

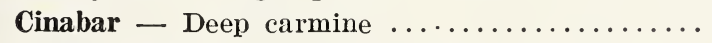

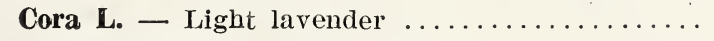




\section{George L. Stillman's Dahlia PRICE LIST}

\section{NEW PAEONY FLOWERED---(Continued)}

92 Curleyeue - Reddish pink ............. 1.00

5.00

184

19 Crazy Mike - Pure white

5.00

25.00

59 Charlene - Light salmon

5.00

25.00

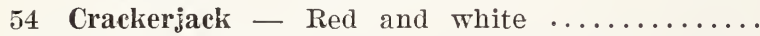

1.00

5.00

.75

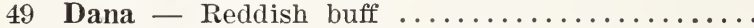

.50

3.75

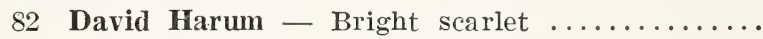

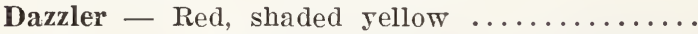

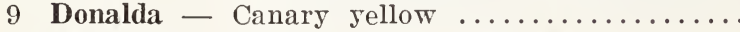

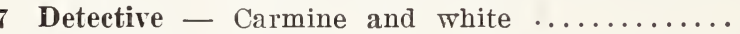

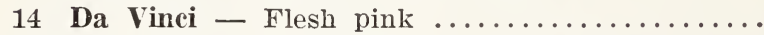

219 Dorothy Dewey - Yellow, shaded pink ......

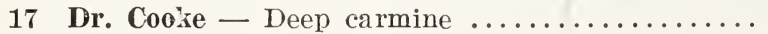

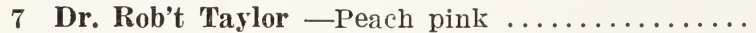

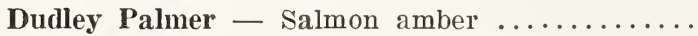

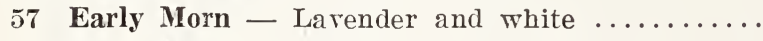

24 Earle Williams - Ruby and white ..........

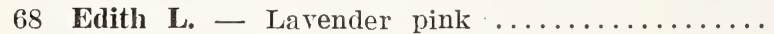

173 Eclipse - Magenta and white ............ 2.00

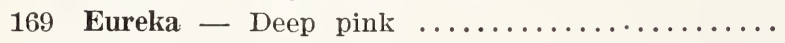

74 Evening Star - Red, tipped yellow ..........

21-Evening Bell — Light salmon ............

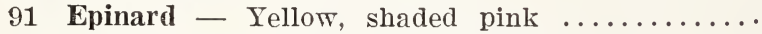

105 Firegirl - Scarlet, tipped yellow ...........

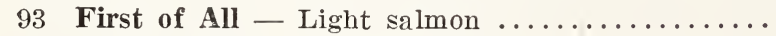

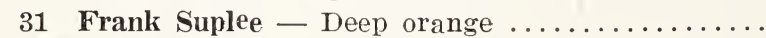

252 Frieda Hempel - Light pink .............

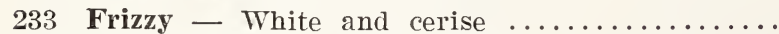

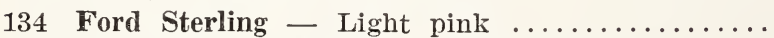

149 Florence $\mathbf{C}$ - Magenta, clouded white .......

91 F. X. Bushman - Pink and yellow .........

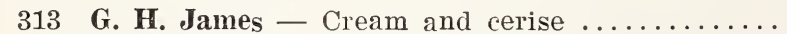

122 Gentleman Sir - Magenta and white .........

59 Golden Feather - Yellow, shaded pink .......

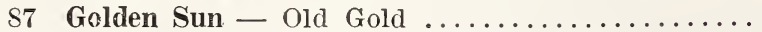

25 Golden Rays - Salmon, shaded yellow .......

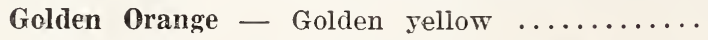

6 Grand Malta - Carmine, tipped yellow ....... 


\section{George L. Stillman's Dahlia PRICE LIST}

No. of

Clumps

NAME - CLASS - DESCRIPTION

Price

Price

Total No. per Bulb perDozen Divisions

\section{NEW PAEONY FLOWERED---(Continued)}

134 221

\section{8}

Harry McCoy - Canary yellow

$$
\text { ................... }
$$

\subsection{0}

2.00

Hay Day - Bright carmine ............. 2.00

Helen Louise - Deep butter yellow .........

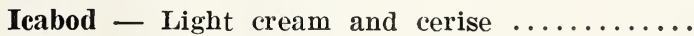

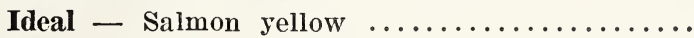

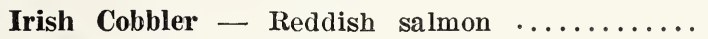

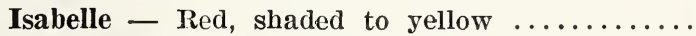

Irva Varrelman - White, shaded light cream ..

J. Austin Shaw - Peach yellow and pink .....

Jane Witherspoon - Carmine, tipped canary ...

Jean Anderson - Salmon buff and yellow .....

Jack Dempsey - Carmine and yellow ........

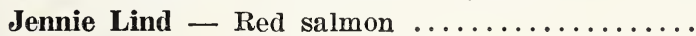

Jim Dandy - Magenta, tipped white .........

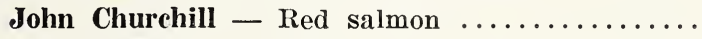

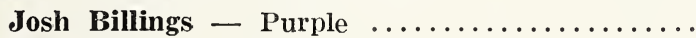

John Jones - Yellow, clouded salmon

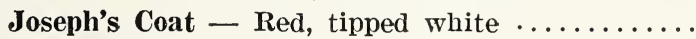

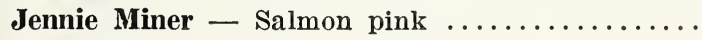

King Saul - Bright carmine $\ldots \ldots \ldots \ldots \ldots \ldots$

Kinney - Pink

Klondyke - Red apricot and yellow

Koman - Yellow and salmon ........

L. Wellington - Deep pink and white ........

Lady Astor - Purple, carmine and white .......

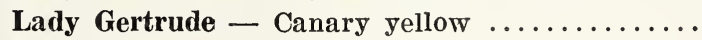

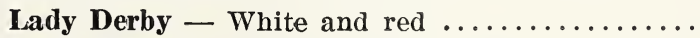

Lady Marie - Yellow, clouded pink ..........

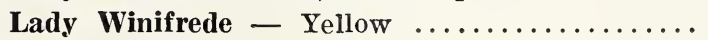

Ladies' Delight -Lavender and pink .........

La La - Light purple $\ldots \ldots \ldots \ldots \ldots \ldots \ldots$

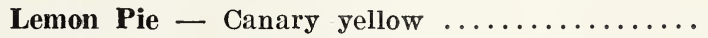

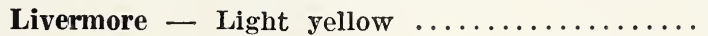

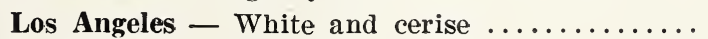

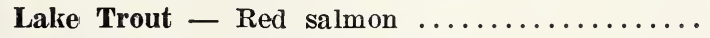

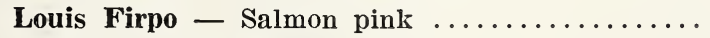

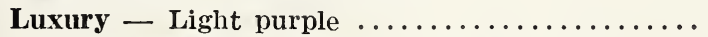

Lord Beaconsfield - Carmine, tipped white ....

Mable Martin - Cerise, clouded white ........

Marguerite Clarke - Cream and cerise ........

Marie Suplee - Butte yellow .............

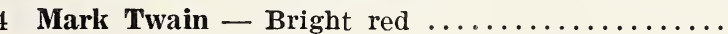

3.00

1.50

3.00

2.00

.50

.50

.75

1.50

1.00

3.00

2.00

.50

1.00

.75

5.00

1.00

5.00

1.50

1.50

1.50

3.00

5.00

3.00

5.00

5.00

5.00

2.00

3.00

2.00

1.00

1.50

1.00

2.50

5.00

3.00

3.00

1.00

2.00

2.00

1.50
10.00

10.00

15.00

8.00

15.00

10.00

2.50

8.00

3.75

8.00

5.00

15.00

10.00

2.50

5.00

3.75

25.00

5.00

25.00

8.00

8.00

8.00

15.00

25.00

15.00

25.00

25.00

25.00

10.00

15.00

10.00

5.00

8.00

5.00

12.50

25.00

15.00

15.00

5.00

10.00

10.00

8.00
268

442

16

84

96

110

32

90

180

1750

168

86

18

108

12

550

90

10

140

56

188

348

290

102 


\section{George L. Stillman's Dahlia PRICE LIST}

No. of
Clumps

$\begin{array}{cc}\text { Price } & \text { Price } \\ \text { per Bulb } & \text { per Dozen }\end{array}$

Total No.

Divisions

\section{NEW PAEONY FLOWERED---(Continued)}

19

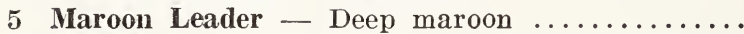

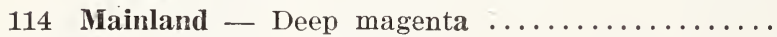

435

3

77

38

169

221

73

185

64

4

65

95

Marke
Maroo
Mainl

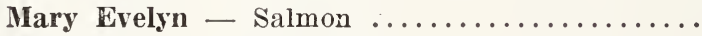

Mayflower - Deep orange $\ldots \ldots \ldots \ldots \ldots \ldots$

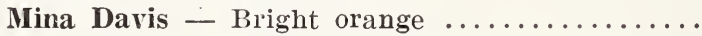

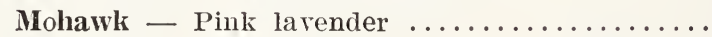

Ming Toy - Rose pink

Minnie Foster - Tan or straw color ..........

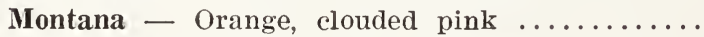

Mock Orange - Yellow, tinted pink ..........

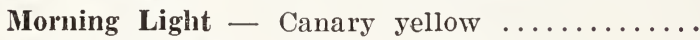

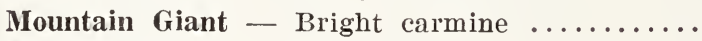

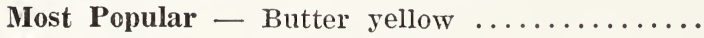

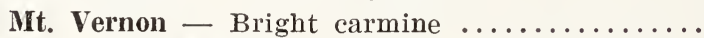

Mrs. A. J. Moulton - Orange, shaded yellow ..

Mrs. E. J. Layng - Pink and yeliow .........

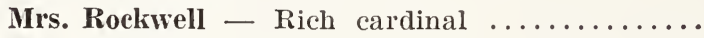

Mrs. Wm. F. Lyons - Yellow, shaded pink ....

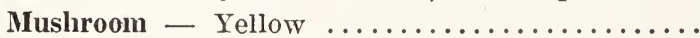

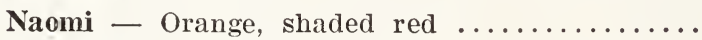

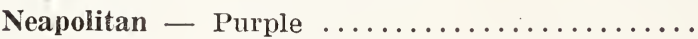

Nora Belle - Yellow clouded cerise..........

Nature's Gift - Yellow, shaded pink .........

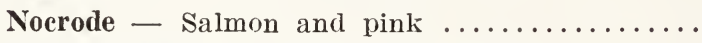

old Homestead - Reddish peach pink .........

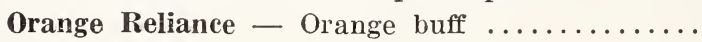

Ostrich Plume - Yellow clouded red .........

Orange Bride - Orange, salmon and pink .....

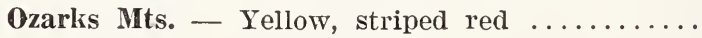

Over the Top - Rose Lavender .............

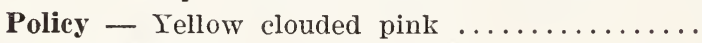

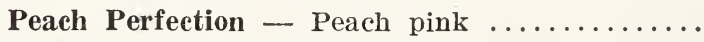

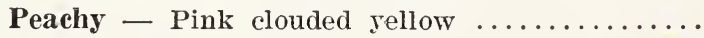

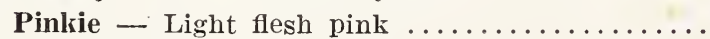

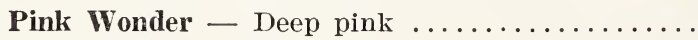

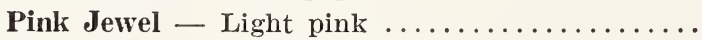

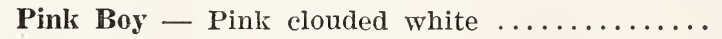

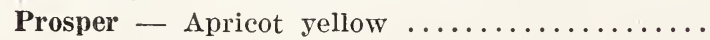

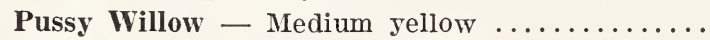

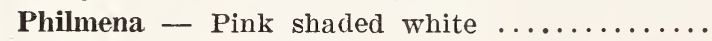

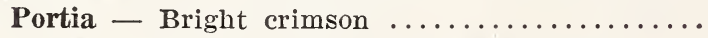

Queen of Pinks - Pink, overcast lavender ......
1.00

.75

1.50

3.00

5.00

1.50

1.00

1.50

2.00

1.50

1.00

1.00

3.00

3.00

2.00

2.00

2.00

.50

3.00

3.00

3.00

2.50

2.00

2.00

1.50

2.00

1.50

1.00

2.50

5.00

1.50

2.00

2.00

3.00

2.00

2.00

2.00

3.00

.75

1.00

1.00

5.00

2.00

\subsection{0}

38

3.75

8.00

228

15.00

25.00

8.00

5.00

8.00

10.00

8.00

5.00

5.00

15.00

15.00

870

6

154

76

338

442

146

370

128

8

130

190

174

24

206

2.50

44

66

76

200

12.50

8

164

38

174

10.00

140

5.00

60

48

136

12.50

148

8.00 


\section{George L. Stillman's Dahlia PRICE LIS'T}

\section{NEW PAEONY FLOWERED---(Continued)}

Radiant Charming - White clouded pink .....

Reba Crawford - Salmon, tipped white .......

Red Eagle - Rich carmine

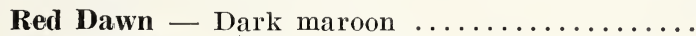

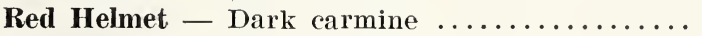

Razzle Dazzle - Carmine, tipped white .......

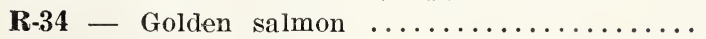

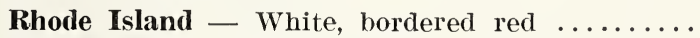

Rocky Mts. - Red salmon ............. 10.00

Rose Dream - Peach salmon ............ 3.00

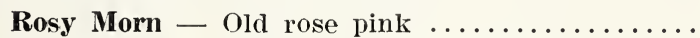

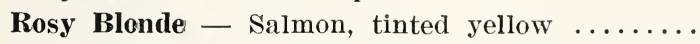

Ruth Thompson - Salmon and yellow ........

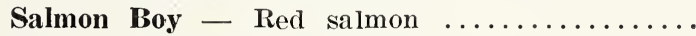

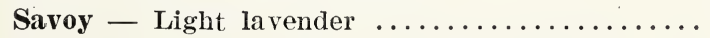

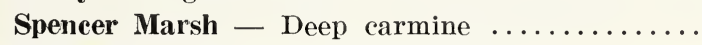

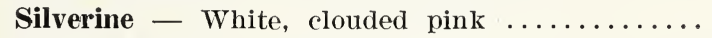

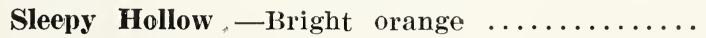

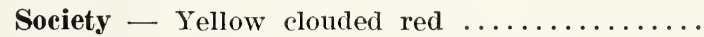

Stillman's Red Cross - Bright carmine .......

Stately Lady - Yellow shaded salmon ........

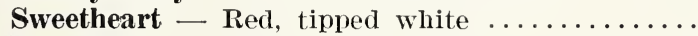

Sweet Lady - Pink clouded white ..........

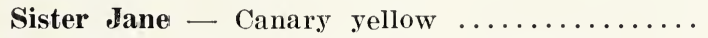

Sunflower Gem - Cerise pink and white .....

The Richest Prize - Lavender pink .........

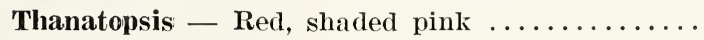

The Colored Jockey - Maroon $\ldots \ldots \ldots \ldots \ldots$

The Old Home - Strawberry $\ldots \ldots \ldots \ldots \ldots \ldots$

The Royal Mayor - Butter yellow ..........

The Gay Dancer - Carmine, tipped white ....

The Heroine - Magenta, clouded pink .......

The Lilac Prince - Lilac, shaded lighter ......

The Billionaire - Golden orange ..........

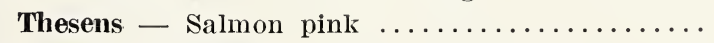

Thomy Thompson - Golden salmon .........

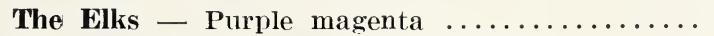

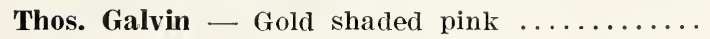

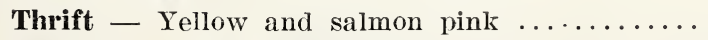

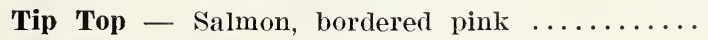

Verna Thompson - White clouded cerise ......

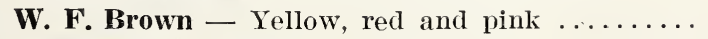

Westover - Yellow

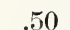

2.50

384

5.00

$2.00 \quad 10.00$

1.00

1.00

2.00

1.50

3.00

1.00

1.00

3.00

3.00

2.50

5.00

.75

2.00

1.50

1.00

5.00

2.00

2.00

1.00

1.00

10.00

1.00

2.00

5.00

3.00

5.00

3.00

5.00

2.00

5.00

2.00

10.00

1.50

2.00

2.00

1.00

2.00

1.00
25.00

5.00

5.00

10.00

8.00

15.00

50.00

15.00

5.00

5.00

15.00

15.00 


\section{George I. Stillman's Dahlia PRICE LIST}

No. of

Clumps

NAME - CLASS - DESCRIPTION

$\begin{array}{ccc}\text { Price } & \text { Price } & \text { Total No. } \\ \text { per Bulb } & \text { per Dozen } & \text { Divisions }\end{array}$

\section{NEW PAEONY FLOWERED---(Continued)}

\begin{tabular}{|c|c|c|c|c|}
\hline 174 & W. T. MacFarlane - Cardinal clouded yellow .. & 1.00 & 5.00 & 348 \\
\hline 44 & Westerly - old rose, shaded pink ......... & 2.00 & 10.00 & 88 \\
\hline 39 & White Cap - White $\ldots \ldots \ldots \ldots \ldots \ldots \ldots$ & 1.00 & 5.00 & 78 \\
\hline 3 & White Climax - White $\ldots \ldots \ldots \ldots \ldots \ldots$ & 1.00 & 5.00 & 6 \\
\hline 121 & White Dove - White, shaded cream ......... & .75 & 3.75 & 242 \\
\hline 159 & White Navy - Pure white .............. & 2.00 & 10.00 & 318 \\
\hline 244 & White Pearl - White $\ldots \ldots \ldots \ldots \ldots \ldots$ & 1.50 & 8.00 & 488 \\
\hline 83 & 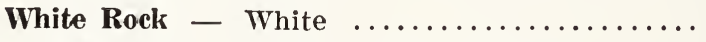 & 1.50 & 8.00 & 166 \\
\hline 182 & Winter Queen - Pink, shaded lavender ...... & 3.00 & 15.00 & 364 \\
\hline 220 & Wyoming - Light pink and yellow ......... & 1.00 & 5.00 & 440 \\
\hline 61 & Yellow Mountain - Canary yellow .. & 5.00 & 25.00 & 122 \\
\hline 125 & Yellowstone - Yellow $\ldots \ldots \ldots \ldots \ldots \ldots$ & 3.00 & 15.00 & 250 \\
\hline 7 & Ýorkshire Hero - Orange and pink ...... & 2.00 & 10.00 & 14 \\
\hline
\end{tabular}

\section{PAEONY FLOWERED---OLD STANDARD VARIETIES}

12 Annie Slocombe - Canary yellow .......... 1.00

9 Bertha Van Suttner - Pink shaded yellow .... 1.00

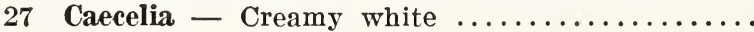

362 Dr. Peary - Dark mahogany red ..........

23

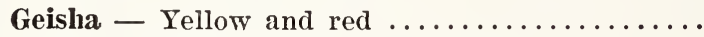

Isaquah - Light purple .............. $\quad .50$

Miss Adelaide Buttle - Lemon yellow .........

P. W. Jannsen - Salmon shaded yellow ......

\section{NEW CACTUS PAEONY}

47 Allie Haley - Bright carmine ........... 1.50 


\section{George L. Stillman's Dahlia PRICE LIST}

No. of

\section{NEW CACTUS PAEONY---(Continued)}

421 Cherry Tree - Cherry pink ........... 1.00

$\begin{array}{rrr}1.00 & 5.00 & 842 \\ 1.00 & 5.00 & 56 \\ 1.50 & 8.00 & 156 \\ 1.50 & 8.00 & 276 \\ 5.00 & 25.00 & 6 \\ 5.00 & 25.00 & 74 \\ 1.50 & 8.00 & 116 \\ 1.50 & 8.00 & 1018 \\ 1.50 & 8.00 & 124 \\ 1.50 & 8.00 & 28 \\ 3.00 & 15.00 & 8 \\ 1.50 & 8.00 & 278 \\ 1.50 & 8.00 & 52 \\ 2.00 & 10.00 & 42 \\ 1.00 & 5.00 & 4 \\ .50 & 2.50 & 190 \\ 1.50 & 8.00 & 22 \\ 3.00 & 15.00 & 8\end{array}$

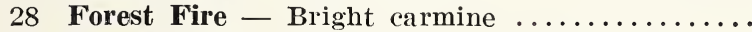

78

138

509

62

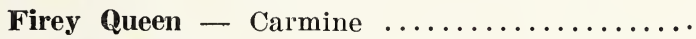

Harold Darling - Light orange ...............

Lady Finger - Orange, tipped white $\ldots \ldots \ldots \ldots$

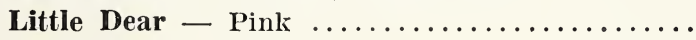

Lusitania - Rose pink

Mrs. Stillman - Pink ...................

Morning Best - Light pink $\ldots \ldots \ldots \ldots \ldots \ldots$

Nature's Crown - Salmon pink .............

Nettie C. - Yellow salmon ................

Pink Crown - Flesh pink ................

Pink Ribbon - Salmon pink and yellow .......

The Gay Boy - Light scarlet ..............

Velvet Spider - Deep carmine ...............

Wild Bill - Bright red ...................

W. W. Kennedy - Canary yellow .............

The Jewel Case - Peach pink

3.00

\section{NEW SEMI-PAEONY OR DUPLEX}

38 Adelaide - Red, tipped yellow ........... .50

26 Altadena - Cerise and white $\ldots \ldots \ldots \ldots \ldots \ldots$

10 Autumn Sun - Salmon yellow and pink .......

110 Banner Girl - White, clouded yellow ..........

100 Black Servant - Magenta, almost black ......

17 Black Valentine - Dark red ...............

8 Belle of the Ball - Cerise, yellow and white ...

79 Ren Hur - Scarlet, slightly yellow ..........

35 Bulah - White, tinted pink ...............

94 Buster Brown - Yellow, streaked red ........

90 Camille - Purple pink .................

150 Cygnet - Deep purple $\ldots \ldots \ldots \ldots \ldots \ldots \ldots$

Hubby Hapgood - Cerise, shaded white ..... $\quad .50$

James Gray - Magenta ................. $\quad .50$

Jennifer Hadden - Cerise and white ....... .50

Koros - Bright maroon .............. 1.00

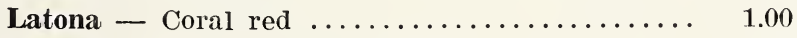

Lenore - White, clouded cerise .......... $\quad .50$ 


\section{George L. Stillman's Dahlia PRICE LIS'T}

\begin{tabular}{|c|c|c|}
\hline $\begin{array}{l}\text { No. of } \\
\text { Clumps }\end{array}$ & NAME - CLASS - DESCRIPTION & $\begin{array}{c}\text { Price } \\
\text { per Bulb }\end{array}$ \\
\hline
\end{tabular}

\section{NEW SEMI-PAEONY---(Continued)}

42 Mona - Crushed strawberrry ......................

14 Marigold - Light fawn ............... 1.00

23 Mrs. Wiggs - Salmon, shaded to canary ......

139 My Girl Sally - Cherry red and yellow ........

46 Not-So-Bad - White, bordered with red ......

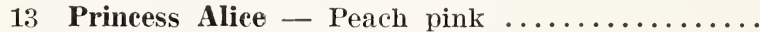

10 Reward - Yellow, veined pink ..............

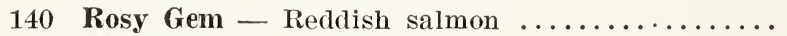

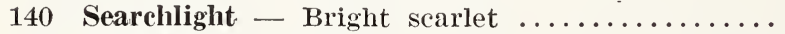

41 Saffron - Peach pink .................. 1.00

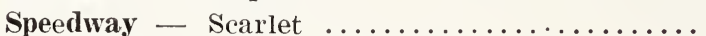

Stella Razets - Cerise veined white ..........

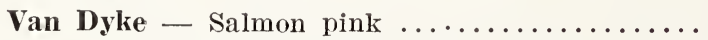

\section{NEW DECORATIVES}

B. P. O

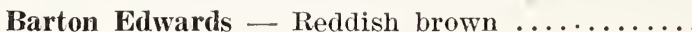

3.00

15.00

106

3.00

15.00

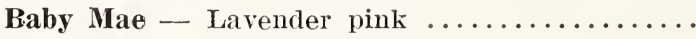

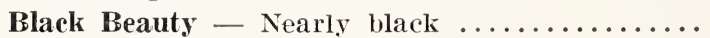

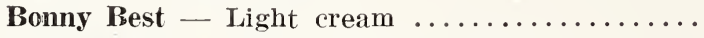

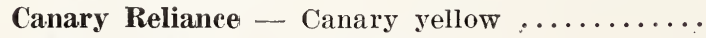




\section{George L. Stillman's Dahlia PRICE LIST}

No. of Clumps

NAME - CLASS - DESCRIPTION $\begin{array}{ccc}\text { Price } & \text { Price } & \text { Total No. } \\ \text { per Bulb } & \text { per Dozen } & \text { Divisions }\end{array}$

Price

Total No.

\section{NEW DECORATIVES---(Continued)}

180

44

112

86

164

253

5

483

38

181 None So Pretty - White, overcast pink .......

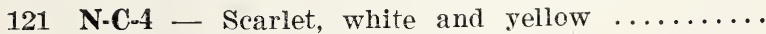

E. J. Pease - Red, veined yellow Florence Nightingale-Yellow, veined salmon Florence Griswold - White, stained red .......

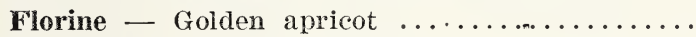

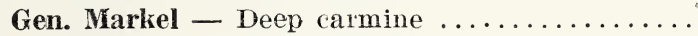

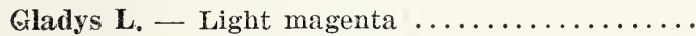

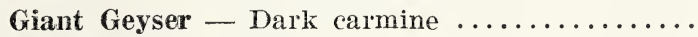

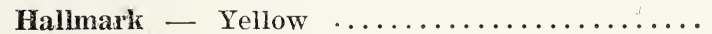

Hocus Pocus - Yellow salmon .............

Immensity - Orange $\ldots \ldots \ldots \ldots \ldots \ldots \ldots \ldots$

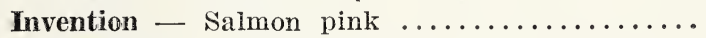

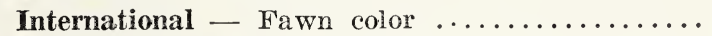

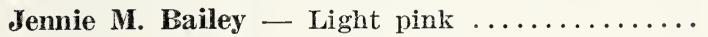

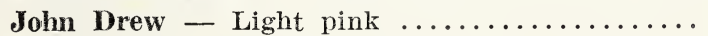

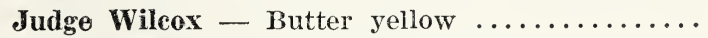

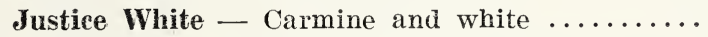

Jim Henry - Salmon, clouded yellow .........

King of the Garden - Pink and yellow .......

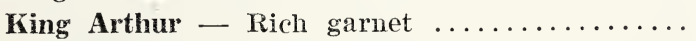

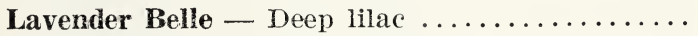

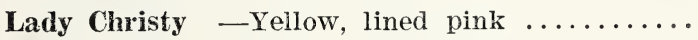

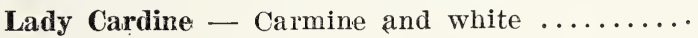

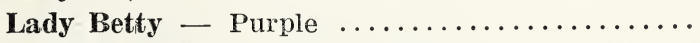

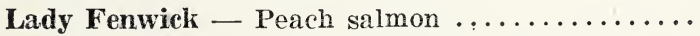

Lady Retta - Pink, lavender tint $\ldots \ldots \ldots \ldots \ldots$

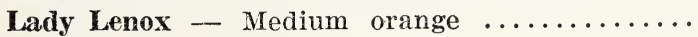

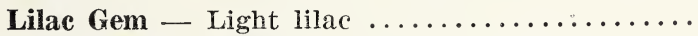

Mary Sefton Thomas - Carary yellow .........

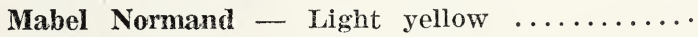

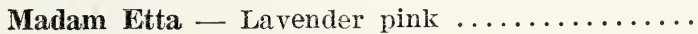

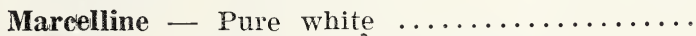

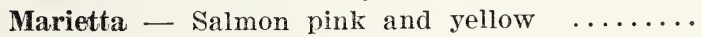

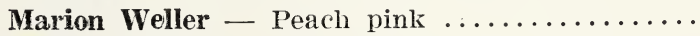

Marilyn Miller - Salmon, shaded yellow .......

Meadow Vale - Canary yellow and white .....

Millionaire - Lavender . . . . . . . . . . . .

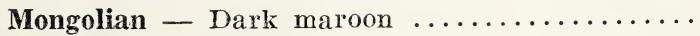

Mr. Layng - Deep pink . . . . . . . . . . . .

Mrs. A. H. Smith - Canary yellow ........

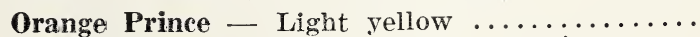

Our Country - Purple, tipped white .......
1.00

5.00

3.00

2.00

15.00

10.00

2.50

1.00

.50

5.00

1.50

3.00

2.00

5.00

3.00

5.00

2.00

3.00

2.50

2.00

5.00

1.00

3.00

3.00

2.00

1.50

2.00

1.00

3.00

1.50

2.00

1.00

2.00

1.00

5.00

3.00

1.50

1.50

1.00

.25

2.00

2.00

.75

2.00

2.00

5.00

15.00

10.00
360

88

224

172

328

506

10

966

76

110

4

418

130

422

48

100

216

636

58

90

32

228

52

114

80

128

8.00

5.00

10.00

5.00

25.00

15.00

8.00

8.00

5.00

1.25

10.00

10.00

3.75

10.00

80

64

356

62

192

14

28

112

220

1300

62

222 


\section{George L. Stillman's Dahlia PRICE LIST}

No. of NAME - CLASS - DESCRIPTION

$\begin{array}{ccc}\text { Price } & \text { Price } & \text { Total No. } \\ \text { per Bulb } & \text { per Dozen } & \text { Divisions }\end{array}$

Clumps

per Bulb per Dozen

\section{NEW DECORATIVES---(Continued)}

32

16

124

170

122

259

125

108

6

8

541

23

23

Oweenee - Cerise and white

1.00

5.00

64

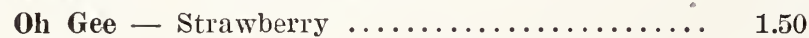

8.00

2.00

10.00

32

Perfect Beauty - Scarlet, tipped white ......

5.00

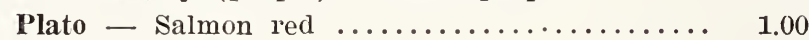

25.00

248

Pride of the West - Golden orange $\ldots \ldots \ldots \ldots$

.75

5.00

2.00

3.75

Pride of the Garden - Yellow and white ......

5.00

10.00

340

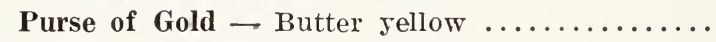

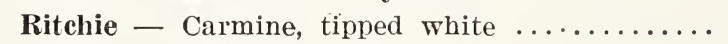

5.00

Richest of All - Rich red ............. 1.50

25.00

25.00

2.00

8.00

244

518

250

Rob't 0. Fletcher - White and red ...........

5.00

10.00

216

Sierra Nevada Mts. - Salmon pink and yellow .

2.00

Senator Spencer - Canary yellow .......... 3.00

The World Court - Yellow, pink and fawn ... 25.00

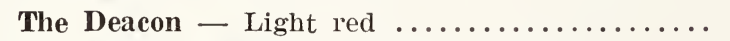

1.50

The World Traveler - Purple magenta .......

5.00

1.50

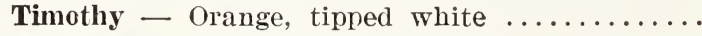

3.00

1.50

2.00

25.00

10.00

15.00

125.00

8.00

25.00

8.00

15.00

8.00

10.00

15.00

75.00

\section{DECORATIVES---OLD STANDARD VARIETIES}

42

300

Ardene - Deep yellow ................ 2.00

Atalanta - White, shaded larender ....... .25 


\section{George L. Stullman's Dahlia PRICE LIST}

\section{DECORATIVES---OLD STANDARD VARIETIES---(Continued)}

46

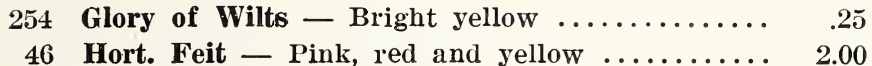

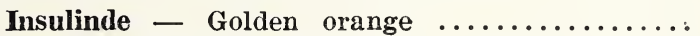

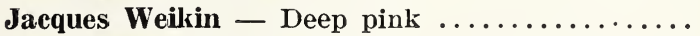

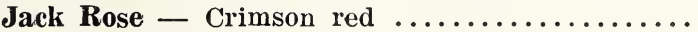
Kalaekahio - Pink, shaded yellow ........... Mons. Burel - Yellow, shaded lighter ......... Judge Marean - Salmon pink, red and gold ... Le Grand Manitou - Pink, spotted red ........

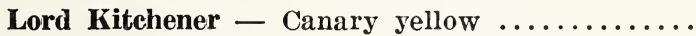
Mrs. I. Dev. Warner - Lavender purple .......

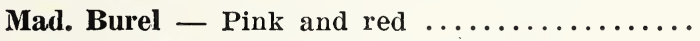
Mad. A. Nonin - White and lavender ......... Mad. L. Tillier - Salmon, blended yellow ....

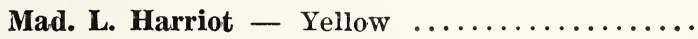

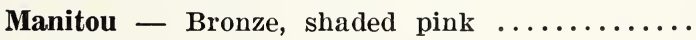

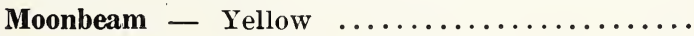

Mrs. Roosevelt - Pink and rose $\ldots \ldots \ldots \ldots \ldots$

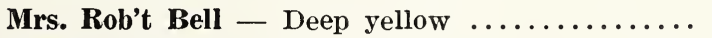

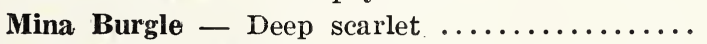

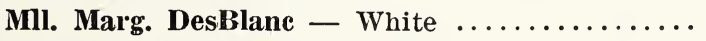

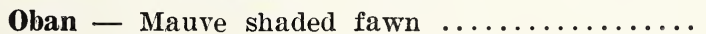

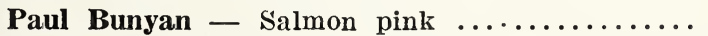

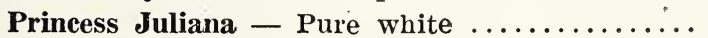
Pride of New Haven - Butter yellow ........ Royal Scarlet - Scarlet $\ldots \ldots \ldots \ldots \ldots \ldots \ldots$

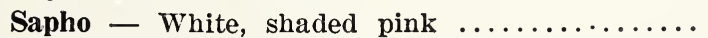

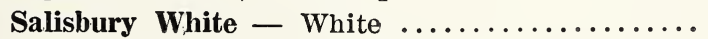

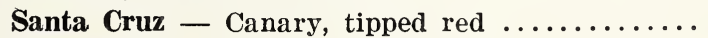

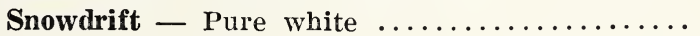

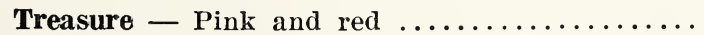
Thompson II - Light red $\ldots \ldots \ldots \ldots \ldots \ldots$

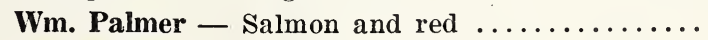
Yellow Colosse - Canary yellow
1.25

10.00

2.50

1.25

1.25

10.00

1.25

25.00

1.25

5.00

5.00

1.25

1.25

1.25

1.25

1.75

2.50

2.50

10.00

2.50

2.50

1.25

2.50

2.50

5.00

1.75

1.25

1.25

5.00

5.00

1.25

1.25

1.75

1.25
508

92

10

62

244

230

46

54

444

176

830

266

268

60

360

54

10

14

122

70

44

210

44

20

50

124

260

58

146 174. . 216

\section{NEW CACTUS}

6 Annabelle (HC) Very deep maroon ......... 10.00 


\section{George L. Stillaman's Dahlia PRICE LIST}

No. of NAYE - CLASS - DESCRIPTION

\section{NEW CACTUS-.-(Continued)}

64 Black Pekin (HC) Nearly black ............. 2.00

10.00

$3.00 \quad 15.00$

85

59

2

51

127

60

111

116

18

9

47

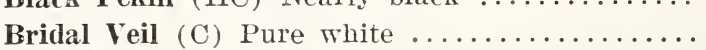

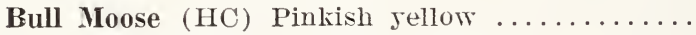

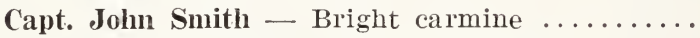

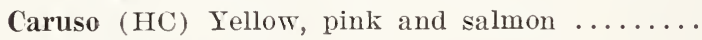

Carrie Hammond (C) Pink, shaded yellow .....

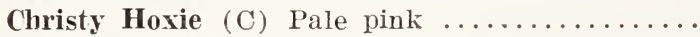

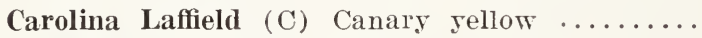

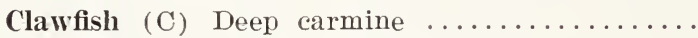

Cuba Ward (C) Carmine, tipped sellow ........

Connecticut College (C) Yellow and salmon ....

Dr. Walter Van Fleet (HC) Deep rose pink ...

Duchess of Wellington (HC) Canary yellow ..

Doris Snow (HC) White, overcast pink .......

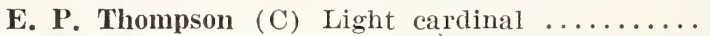

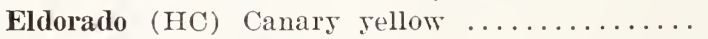

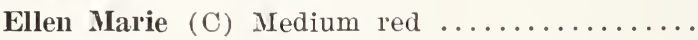

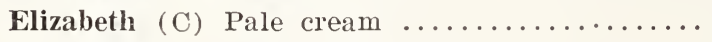

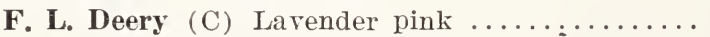

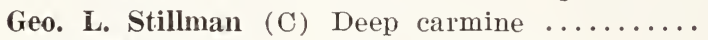

Gilbert McClurg (C) Yellow and salmon .......

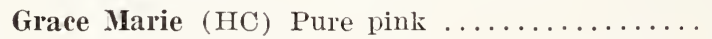

Head of the Nation (C) Purple and magenta ...

Hollyhock (HC) Salmon pink and yellow ......

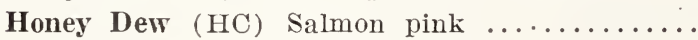

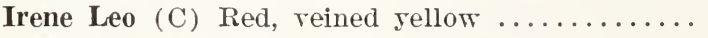

League of Nations (C) Canary sellow .........

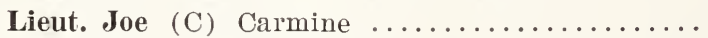

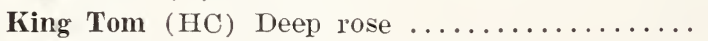

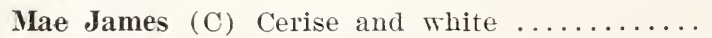

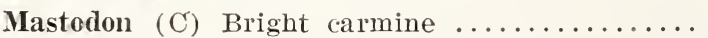

Mrs. Aaron Ward (C) Light red and yellow ....

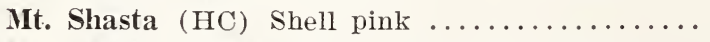

Mrs. M. E. Foster (HC) Yellow, stirped red ....

Mrs. Marshall (C) Yellow, overcast pink ......

Miss Hannon (HC) Salmon pink and yellow ...

New England Beauty (HC) Carmine red .......

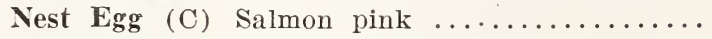

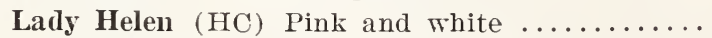

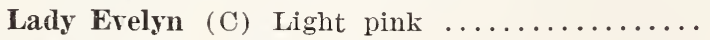

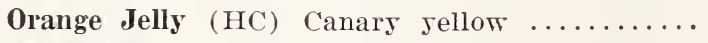

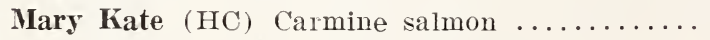

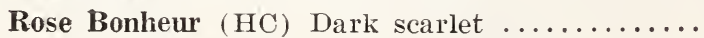

2.00

5.00

5.00

.50

1.00

1.00

1.00

1.00

5.00

10.00

50.00

3.00

2.00

1.50

5.00

2.00

1.00

3.00

5.00

3.00

25.00

2.00

2.00

5.00

5.00

1.00

2.00

1.00

3.00

1.50

2.00

25.00

10.00

10.00

5.00

1.50

3.00

3.00

2.00

15.00

1.00
128

170

118

25.00

25.00

2.50

5.00

5.00

5.00

5.00

25.00

50.00

250.00

15.00

10.00

8.00

25.00

10.00

5.00

15.00

25.00

15.00

125.00

10.00

10.00

25.00

25.00

5.00

10.00

5.00

15.00

8.00

10.00

125.00

50.00

50.00

25.00

8.00

15.00

15.00

10.00

75.00

5.00
4

114

254

120

222

232

36

18

94

56

48

18

100

6

186

226

108

58

110

280

96

286

23

210

86

28

206

128

454

128 


\section{George L. Stilluman's Dahlia PRICE LIS'T}

\section{NEW CACTUS---(Continued)}

7
St. Lawrence (HC) Butter yellow

\subsection{0}

25.00

$3.00 \quad 15.00$

2.00

3.00

3.00

2.00

3.00

5.00

15.00

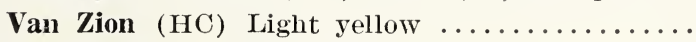

Yuletide (C) Pure white

\section{CACTUS---OLD STANDARD VARIETIES}

\begin{tabular}{|c|c|c|c|c|}
\hline 26 & Advance - Light red & .25 & 1.25 & 52 \\
\hline 42 & Alexander - Crimson $\ldots$. & .50 & 2.50 & 84 \\
\hline 2 & Ami Bethier - Light red $\ldots \ldots \ldots \ldots \ldots \ldots$ & .25 & 1.25 & 4 \\
\hline 9 & Arc-en-Ciel - Pink and white $\ldots \ldots \ldots \ldots \ldots$ & .25 & 1.25 & 18 \\
\hline 43 & Attraction - Lavender with pink sheen ...... & .50 & 2.50 & 86 \\
\hline 23 & Adrian Knox - Bright vermillion .......... & .35 & 1.75 & 16 \\
\hline 12 & 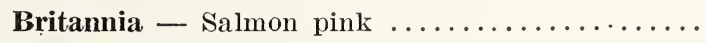 & .50 & 2.50 & 24 \\
\hline 28 & Cannel's Crest - Deep crimson ............ & .25 & 1.25 & 66 \\
\hline 3 & Cardinal - Cardinal red $\ldots \ldots \ldots \ldots \ldots \ldots$ & .25 & 1.25 & \\
\hline 61 & Bizine - White shaded crimson $\ldots \ldots \ldots \ldots \ldots$ & .50 & 2.50 & 122 \\
\hline 55 & Chastity - Pale pink $\ldots \ldots \ldots \ldots \ldots \ldots$ & .50 & 2.50 & 10 \\
\hline 24 & Citizen - Maroon, shaded purple $\ldots \ldots \ldots \ldots$ & .25 & 1.25 & 48 \\
\hline 75 & Comte deRiberia - Yellow and lilac ........ & .35 & 1.75 & 150 \\
\hline 11 & Count. Malmesbury - Pink and white ....... & .25 & 1.25 & \\
\hline 33 & Country Girl - Yellow and orange .......... & .25 & 1.25 & \\
\hline 142 & Cycle - Reddish pink and white........... & .25 & 1.25 & 284 \\
\hline 16 & Coun. Lonsdale - Red and salmon ........ & .25 & 1.25 & \\
\hline 11 & 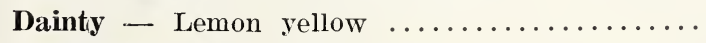 & .25 & 1.25 & \\
\hline 62 & Daisy Stapless - Mauve pink $\ldots \ldots \ldots \ldots \ldots$ & .25 & 1.25 & \\
\hline 2 & Delight - Pink and yellow ............. & .25 & 1.25 & \\
\hline 5 & Dorothy Vernon - Strawberry $\ldots \ldots \ldots \ldots \ldots$ & .25 & 1.25 & \\
\hline 82 & Dr. Mene - Amber and yellow $\ldots \ldots \ldots \ldots \ldots$ & .25 & 1.25 & 164 \\
\hline 8 & Dreadnaught - Wine color $\ldots \ldots \ldots \ldots \ldots$ & .25 & 1.25 & \\
\hline
\end{tabular}




\section{George L. Stillman's Dahlia PRICE LIST}

No. of

Clumps TAME - CLASS - DESCRIPTION

$\underset{\text { per Bulbe }}{\text { Price }}$

Price

Total No. per Bulb perDozen Divisions

\section{CACTUS--.OLD STANDARD VARIETIES---(Continued)}

3 Delicatissima - White and lilac rose ..........

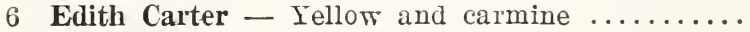

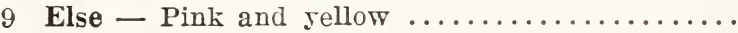

53 Emperor - Plum color and sellow ...........

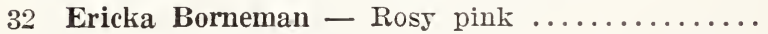

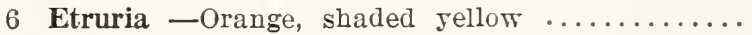

23 Etend. de Lyon - Carmine blended purple ......

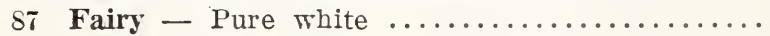

165 Fantassima - Salmon, pink and larender ....

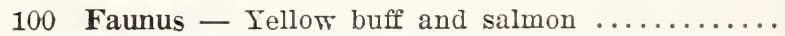

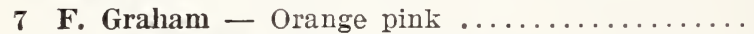

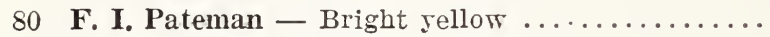

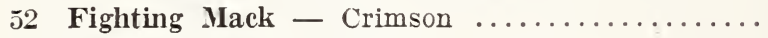

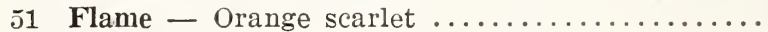

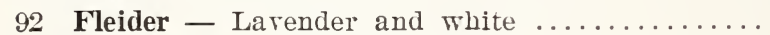

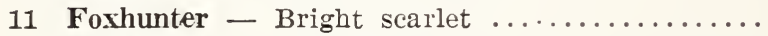

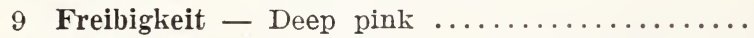

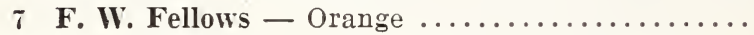

36 Gen. Buller - Crimson tipped white .......

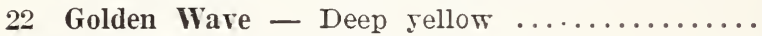

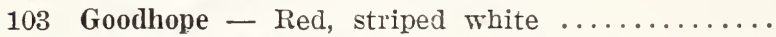

24 Ingleboro, Edgeland - Red and sellow .........

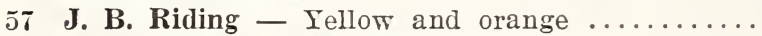

H. W. Sillem - Vermillion scarlet ..........

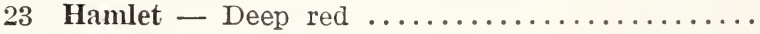

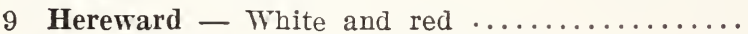

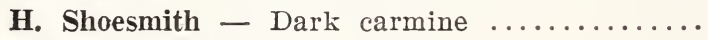

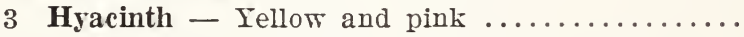

13 H. G. Alford - Yellow, amber and pink .....

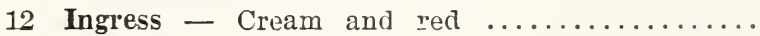

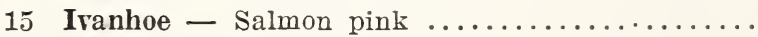

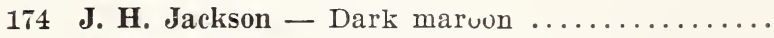

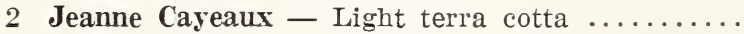

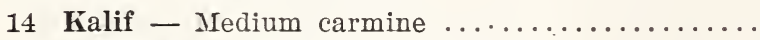

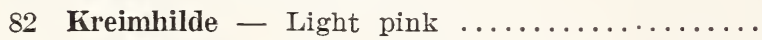

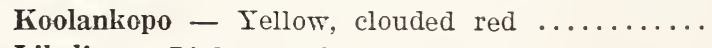

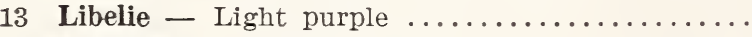

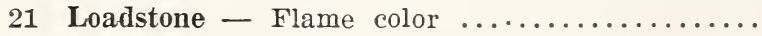




\section{George L. Stillman's Dahlia PRICE LIST}

No. of
Clumps NAME - CLASS - DESCRIPTION

Price

Price

Total No.

per Bulb

per Dozen

Divisions

\section{CACTUS---OLD STANDARD VARIETIES---(Continued)}

6 Lucio - Yellow and white .............. 25

27 Marg. Bouchon — Light pink .............

15 Mephistopheles - Vivid scarlet ............ .25

28 Mme. Henri Martinet - Salmon pink, shaded yel.

109 Marj. Castleton - Rose pink and white .......

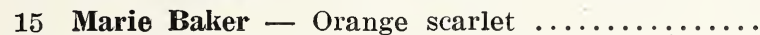

125 Maravigalla - Rose with golden tips .........

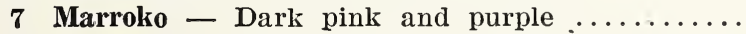

12 Master Carl - Deep yellow .............. $\quad .50$

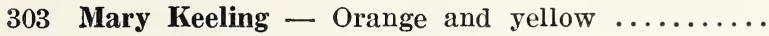

58 Mary Farnsworth - White and light yellow ..

100 Mayfield Beauty - Pink, shaded yellow ......

M. Pierrot - Amber and white $\ldots \ldots \ldots \ldots \ldots$

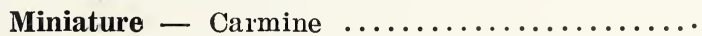

Mirifique - Purplish maroon $\ldots \ldots \ldots \ldots \ldots \ldots$

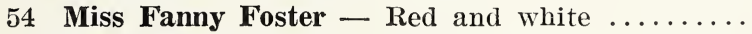

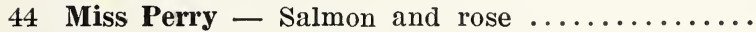

44 Mons. Cazalis - Magenta and white .........

M. Rodger Lecler - Light yellow ...........

100 Mrs. C. H. Breck - Light red and white .....

9 Mrs. D. B. Crane - Pure white ...........

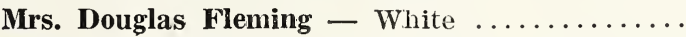

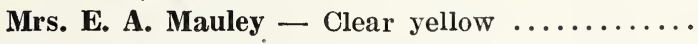

8 Mrs. H. J. Jones - Scarlet and cream ........

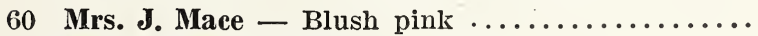

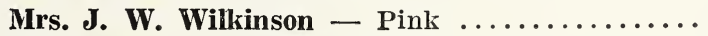

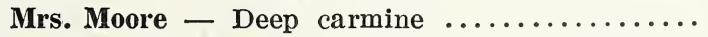

2 Mrs. Reginald Bailey - Deep violet red ......

80 Mrs. Stephen Clarke - Pink, shaded white ...

3 Mrs. T. W. Willis - White and rose liıac .....

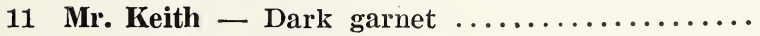

Nahida de Semelle - Light scarlet ..........

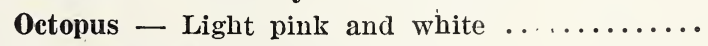

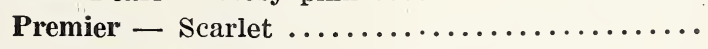




\section{George L. Stillman's Dahlia PRICE LIST}

No. of NAME - CLASS - DESCRIPTION

$\begin{array}{ccc}\text { Price } & \text { Price } & \text { Total No. } \\ \text { per Bulb per Dozen } & \text { Divisions }\end{array}$

\section{CACTUS---OLD STANDARD VARIETIES---(Continued)}

54 Rose Queen - Bright scarlet ............ .50

2.50

108

$5 \quad$ Rupert - Pale amber ................. 1.00

5.00

10

80 Sir A. Lamb - Crimson shaded purple .......

1.25

160

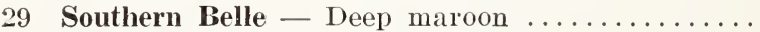

2.50

58

4 Standard Bearer - Light carmine ...........

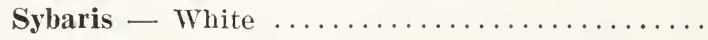

1.25

7 Thuringia - Flame color and orange ........

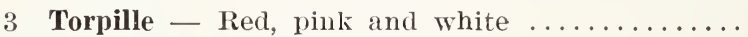

16 Uncle Tom - Dark maroon ..............

71 Una - Pink and white ................

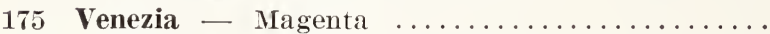

100 Victor Von Scheffel — Pink and white ........

14 Visc. Shearbroke - Red terra cotta .........

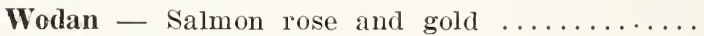

\section{NEW SHOW OR BALL-SHAPE}

25 Andrew Jackson - Deep purple .......... .35

26 Aviator - White, clouded pink ........... 2.00

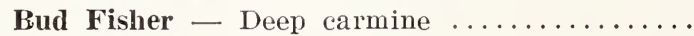

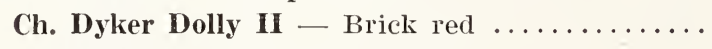

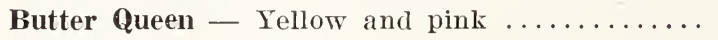

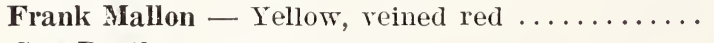

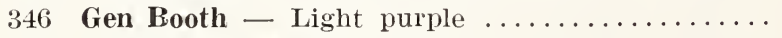

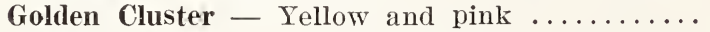

Golden Rod - Yellow and white .......... 2.00

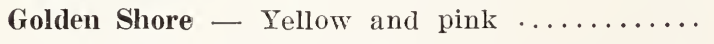

John Stanhope - Red salmon $\ldots \ldots \ldots \ldots \ldots$

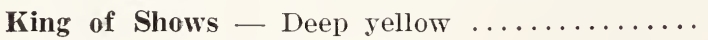

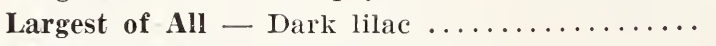

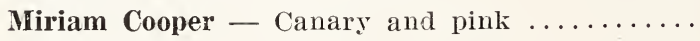

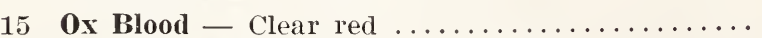




\section{George L. Stillman's Dahlia PRICE LIST}

No. of

\section{NEW SHOW---(Continued)}

77 Red Ball - Medium red ................ 3.00

25.00

728

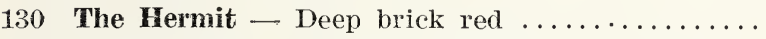

154

27 Tennis Ball - Purple magenta

\section{SHOW--.OLD STANDARD VARIETIES}

85 America - Slate, striped red $\ldots \ldots \ldots \ldots \ldots \ldots \ldots . .25$

5 A. D. Lavoni - Delicate pink ........... $\quad .25$

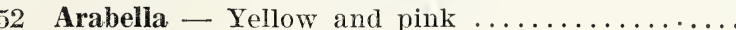

9 Bride - Lavender .....................

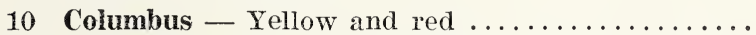

190 Crown Prince - Deep buff $\ldots \ldots \ldots \ldots \ldots \ldots$

34 Cuban Giant - Reddish maroon ............

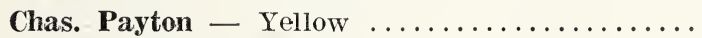

2 Dreer's Yellow - Pure yellow ............ .35

109 Eleanor Ray - Dark red and white ..........

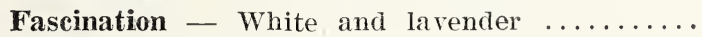

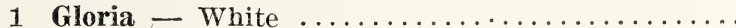

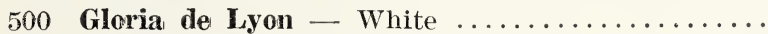

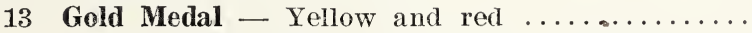

5 Grand Duke Alexis - White and lavender ......

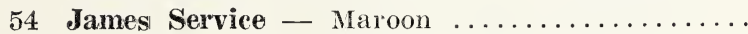

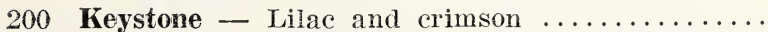

173 Les Armours de Madame - Lavender and red . .

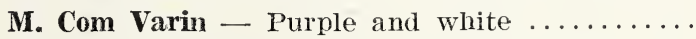

Mme. M. Anagnostaki - White and rose pink ..

9 Mrs. Kendall — White, edged purple ........

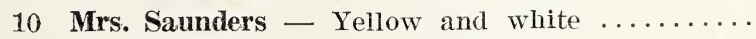

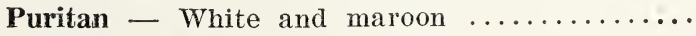

Red Fire - Light red $\ldots \ldots \ldots \ldots \ldots \ldots \ldots$

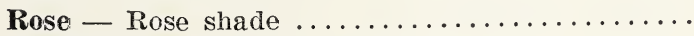

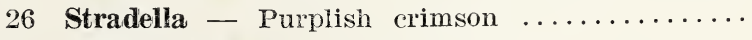

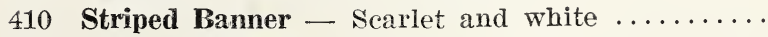




\section{George L. Stilluman's Dahlia PRICE LIS'T}

\section{No. of NAME - CLASS - DESCRIPTION \\ SHOW---OLD STANDARD VARIETIES (Continued)}

$\begin{array}{ccc}\begin{array}{c}\text { Price } \\ \text { per Bulb }\end{array} & \begin{array}{c}\text { Price } \\ \text { per Dozen }\end{array} & \begin{array}{c}\text { Total No. } \\ \text { Divisions }\end{array}\end{array}$

224 Susan - Shell pink ................. .25

90 Tillamook - Pale pink ................ .25

134 Triomphe de Lorraine - Red and salmon .... $\quad .25$

31 Vivian - Magenta and cerise ............ .25

3 W. W. Rawson - White and amethyst ....... .50

1.2562

110 W. H. Williams - Rich scarlet ........... .25

1.25220

83 White Swan - Pure white $\ldots \ldots \ldots \ldots \ldots \ldots \ldots . .25$

$1.25 \quad 166$

\section{NEW COLLARETTES}

105 Annette - Yellow, red and white ........... .25

164 Capt. Roycroft - Maroon, pink and white ....

Color Blender - Yellow and crimson ........ $\mathbf{. 7 5}$

\section{Daisy Smith - Yellow, clouded pink .........}

\section{Grace Loretta - Magenta and white .................................}

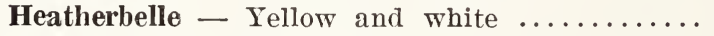

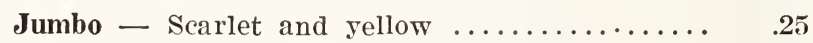

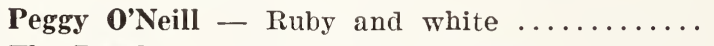

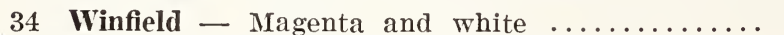

\section{OLD COLLARETTES---OLD STANDARD VARIETIES}

127 A. Mammene - Dark red and white ....... . .25

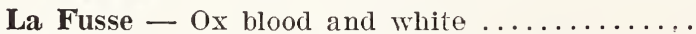

Maurice Riviore - Maroon and white ........

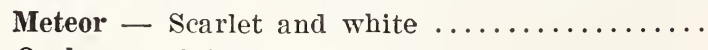

35 Prince Carilie deRohan - Pink and lavender ...

\section{NEW SINGLES}

25 Alameda - Magenta and white ........... .25 


\section{George I. Stillman's Dahlia PRICE LIS'T}

No. of
Clumps NAME - CIASS - DESCRIPTION

$\begin{array}{ccc}\text { Price } & \text { Price } & \text { Total No. } \\ \text { per Bulb } & \text { per Dozen } & \text { Divisions }\end{array}$

\section{NEW SINGLES---(Continued)}

135 Aukum - Light pink ................ ${ }_{.25}$

53 Blushing Boy - Maroon and white ......... 1.00

Chanticleer - Cinnamon and gold .......... $\quad .25$

5.00

106

142

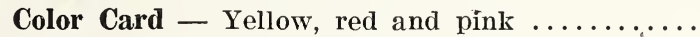

Erebus - Pink and white .............. 1.00

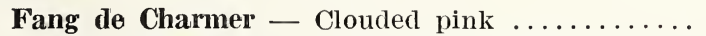

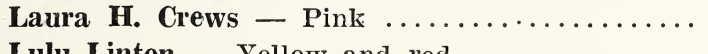

1.00

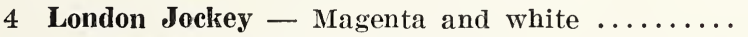

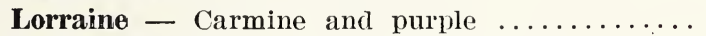

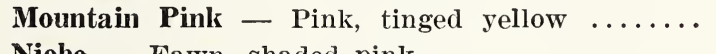

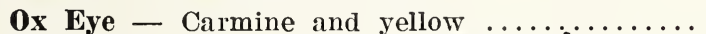

Peggy Lundeen - Cerise, yellow, white ......

Polly - White and $\operatorname{pink} \ldots \ldots \ldots \ldots \ldots$

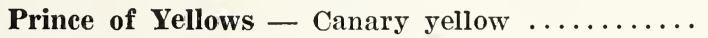

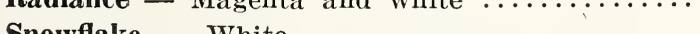

Star of the East - Magenta and white ........

\section{SINGLES---OLD STANDARD VARIETIES}

135 Blush. Century - White and pink ......... .25

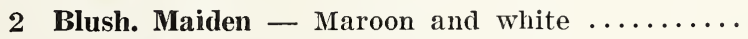

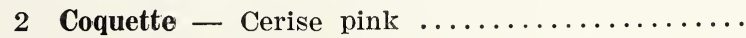

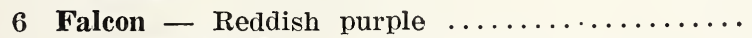

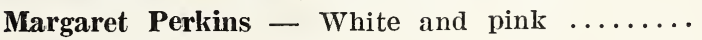

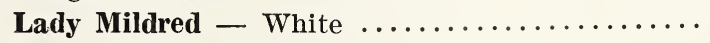




\section{NEW POMPOMS}

7 Wee Willie - Deep orange .............. 1.00

\section{OLD POMPOMS---OLD STANDARD VARIETIES}

19 Ad. Dott - Pink, shaded larender ......... $\quad .20$

3 Ariel - Buff, tinted orange ............ .25

61 Belle of Springfield - Light red ......... .50

5 Blumenfalter - Violet red $\ldots \ldots \ldots \ldots \ldots \ldots \ldots . .25$

78 Brunette - Pink and crimson ........... .50

75 Catharine - Buttercup yellow $\ldots \ldots \ldots \ldots \ldots \ldots \ldots \ldots \ldots \ldots$
10 Codsall Gem - Red and white $\ldots \ldots \ldots \ldots \ldots \ldots$

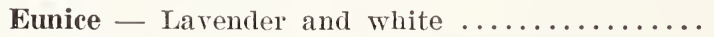

14 Fascination - Pink and lavender, white ......

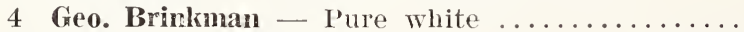

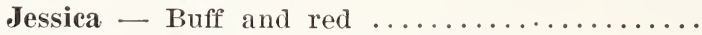

8 Gruss aus Wein - Crushed strawberry ........

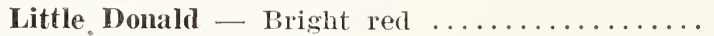

Klein Gretchen - White and crimson .........

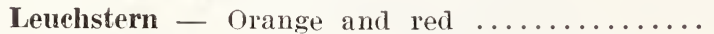

Little Jennie - Primrose yellow ...........

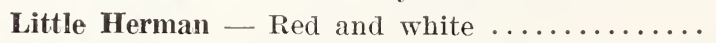

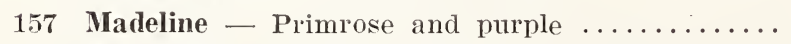

210 Mary Clift - Light maroon $\ldots \ldots \ldots \ldots \ldots \ldots$

190 Marguerite - Deep lilac $\ldots \ldots \ldots \ldots \ldots \ldots \ldots . .25$

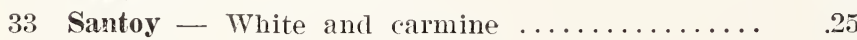

76 Snowelad - Pure white ............... .25

9 Sunset - Deep orange ................ 50

215 Tulla - Wine crimson $\ldots \ldots \ldots \ldots \ldots \ldots \ldots \ldots .25$

167 Zoe - Yellow and white $\ldots \ldots \ldots \ldots \ldots \ldots \ldots . .25$

\section{NEW POMPOM DECORATIVES}

112 Wee Charlie - Bright carmine ............ 1.00 


\section{NEW ORIGINAL INTRODUCTIONS FOR 1927}

5 Apricot (P) Apricot colored ............. 5.00

25.00

10

114

29

39

Big Bouncer $(\mathrm{HC})$ Bright scarlet $\ldots \ldots \ldots \ldots .15 .00$

Banna (HC) Salmon pink .............. 5.00

Conspicuous $(\mathbb{P})$ Kediish salmon .......... 10.00

Crushed Strawberry (D) Strawberry ....... 3.00

Candida

3.50

2.50

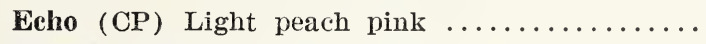

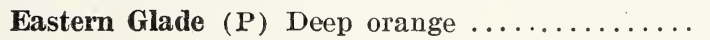

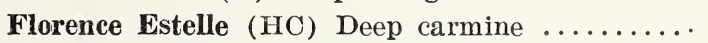

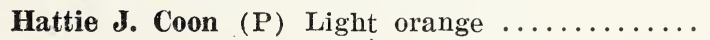

Helen Frances (HC) Canary yellow ......... 10.00

Helen P. Williams (HC) Lavender and white .. 5.00

Hoo Chee (D) Strawberry and yellow ...... 10.00

Gold Bug (HC) Canary yellow ........... 5.00

H. Moors $(P)$ old gold $\ldots \ldots \ldots \ldots \ldots \ldots \ldots$

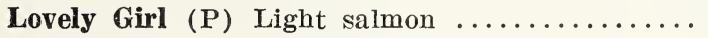

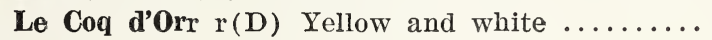

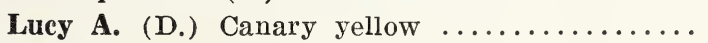

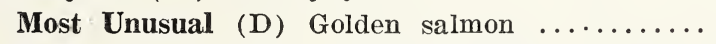

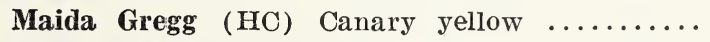

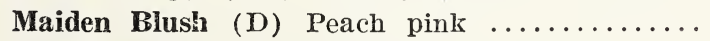

Orangeman $(P)$ Light orange $\ldots \ldots \ldots \ldots \ldots \ldots$

Prince Imperial ( $P$ ) Bright carmine ..........

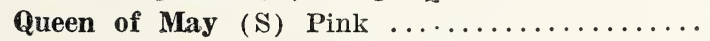

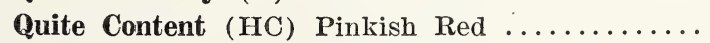

Rhoda Ferguson (HC) Light salmon pink .....

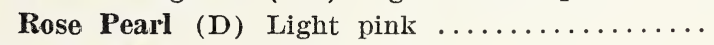

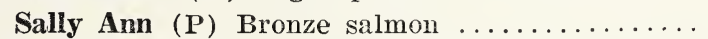

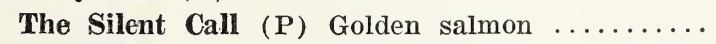

The Trophy (P) Strawberry $\ldots \ldots \ldots \ldots \ldots \ldots$

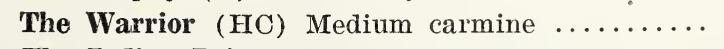

The Indian Princess (D) Salmon pink ........

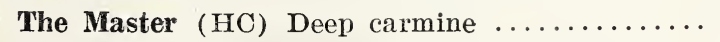

The Glory of Gold (D) Deep butter yellow ....

Katharine Wells (HC) White and light cream .

Yellow Hammer (HC) Canary yellow .........

Welcome Guest (P) Light purple and white ....
5.00

5.00

5.00

5.00

3.00

5.00

3.00

10.00

10.00

3.00

2.50

10.00

5.00

2.50

5.00

3.00

2.50

3.00

2.00

10.00

25.00

10.00

25.00

5.00

5.00

3.00

5.00
75.00

25.00

50.00

15.00

18.00

12.50

25.00

25.00

25.00

50.00

25.00

50.00

25.00

25.00

15.00

25.00

15.00

50.00

50.00

15.00

12.50

50.00

25.00

12.50

25.00

15.00

12.50

15.00

10.00

50.00

125.00

50.00

125.00

25.00

25.00

15.00

25.00
228

58

78

74

56

48

108

96

26

56

94

30

26

126

64

140

56

130

38

36

54

30

70

72

58

50

192

68

40

132

104

40

130

132

54

18

44 


\section{George L. Stilliman's Dahlia PRICE LIST}

\section{SPECIAL OFFERS}

\section{Parcel Post or Express paid by the purchaser. See table below.}

For the benefit of those who are not familiar with the names, colors, and characteristics of the Dahlia, and wish to have the selection made for them, I make the following collections at a special low price to even up stock. They consist of ar. assortment of classes and colors and the entire selection must be left to me.

No. 1 COLLECTION, twelve named varieties, several classes, my own personal selection, no two alike, and each one labeled ............

No. 2 COLLECTION contains twelve fine varieties, my own personal selection, no two alike, and each one labeled. This collection contains

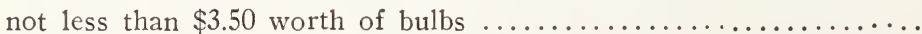

No. 3 COLLECTION contains twelve varieties, or more, of extra nice assorted bulbs very carefully selected, no two alike, all labeled. The Dahlias in this collection amount to not less than $\$ 7.00$ worth .....

No. 4 COLLECTION contains swenty bulbs, without labels, that have become mixed in handling. They are all good varieties, but labels have become detached. The supply for this collection is limited. The quality of bulbs that get into this collection and the present cost of labor makes it positively necessary to make the price of this collection ....................... Postpaid in U. S. A.

\section{Be Sure to Read This}

It has been thoroughly demonstrated that nearly all small shipments of bulbs can be sent by mail and reach their destination quicker than by express.

NOTICE! The postage or express on all special offers must in all cases, except No. 4, be paid by purchaser, and if by mail, postage must be sent with the order or pay C. O. D. charges to destination, which often amounts to more than the postage.

\section{To ascertain the amount of postage to send.}

No. 1, 2 and 3 Collections weigh four pounds or less.

Your postmaster can tell you the cost of any number of pounds from Westerly, R. I. to your post office. Or you can use the following table. If you send more money than is used in postage, the surplus will be returned.

\section{TABLE}

1st \& 2d Zone 3d Zone

1 lb. 5c. 1 lb. $6 \mathrm{c}$.

2 lbs. 6c. 2 lbs. $8 c$.

3 lbs. 7c. 3 lbs. 10c.

4 lbs. 8c. 4 lbs. $12 \mathrm{c}$.

5 lbs. 9c. 5 lbs. $14 \mathrm{c}$.

6 lbs. 10c. 6 lbs. $16 \mathrm{c}$

$\begin{array}{rlr} & 4 \text { th Zone } \\ 1 & \text { lb. } & 7 \mathrm{c} . \\ 2 & \text { lbs. } 11 \mathrm{c} . \\ 3 & \text { lbs. } 15 \mathrm{c} . \\ 4 & \text { lbs. } 19 \mathrm{c} . \\ 5 & \text { lbs. } 23 \mathrm{c} . \\ 6 & \text { lbs. } 27 \mathrm{c} .\end{array}$

5th Zone

6th Zone

$11 \mathrm{~b} . \mathrm{\gamma c}$

2 lbs. $14 \mathrm{c}$.

3 lbs. 20c.

4 lbs. $26 \mathrm{c}$

5 lbs. $32 \mathrm{c}$.

6 lbs. $38 \mathrm{c}$.
1 lb. $9 \mathrm{c}$

2 lbs. $17 \mathrm{c}$.

4 lbs. $33 \mathrm{c}$.

5 lbs. $41 \mathrm{c}$.

6 livs. $49 \mathrm{c}$
7 th Zone

$1 \mathrm{lb}$. 11c

2 lbs. 21c

3 lbs. 31c.

4 lbs. $41 \mathrm{c}$.

5 lbs. 51c.

6 lbs. 61c 8th Zone

1 lb. $12 \mathrm{c}$

2 lbs. 24c.

3 lbs. $36 \mathrm{c}$.

4 lbs. $48 \mathrm{c}$.

5 lbs. 60c.

6 lbs. $72 \mathrm{c}$. 


\section{SEED FIELD CORN}

During the last few years I have taken great pride in producing the best corn for family use that can be grown. I think I have now four varieties that contain all the desirable features that any person could wish for family use. One for corn meal and three for green corn. Prices for selected seed follow.

\section{Stillman's Special Rhode Island Double Cap Corn}

Outer end entirely capped. Butt nearly capped. The best white northern corn grown. The ears are large, butt stems are exceedingly small. The ears are easily husked and broken off. It yields two and three ears to the stalk. The kernels are large and in straight rows. It makes the sweetest and best table meal. The kind that makes the best R. I. Johnny-Cakes. Always get the Stillman strain.

30c. pt. $\$ 3.008$ qts. 50 c. qt. $\$ 5$. half bu.

$\$ 1.50$ four qts.

\section{Corn Meal}

Made from Stillman's Special Rhode Island Double Cap Corn. It's the sweetest and best meal on the market. Ground in an old-fashioned stone "Grist Mill." Fresh every week. Price, 10 lbs. $\$ 1.25$, postuaid in 1 st and $2 \mathrm{~d}$ Zone.

\section{Stillman's Extra Early Giant Sweet Corn}

matures the earliest and has the largest ears of any extra early sweet corn. Nothing like it for the earliest crop.

Selected Seed, 75c. per pint, 40c. per 1-2 pint.

\section{The New Sweet Orange Corn}

Much larger than the Golden Bantam. The sweetest yellow early green corn grown. Grows a medium height and ears profusely.

Selected Seed, 75c. per pint, 40c. per 1-2 pint 


\section{MT. SHASTA SWEET CORN}

The largest and sweetest green corn in the world. This mammoth new corn is in a class by itself. Nothing can beät it for sweetness and its mammoth size.

June 6, 1923. A gentleman in South Carolina wrote me thus: "Please ship me two pounds of seed of your Mt. Shasta corn. I noticed your claim that your Mt. Shasta was the largest and sweetest corn, and I tried a small quantity last year, really thinking you were covering too much territory in your claim. Have decided you knew whereof you spoke. After eating your corn we no longer care for the ordinary sweet corn."

Selected Seed, 75 c. per pint, $40 \mathrm{c}$. per $1-2$ pint

\section{WORM KILLER}

\section{THE WILLIAMS WORM KILLER}

I have taken the agency for this new device for killing the borer inside the Dahlia stalks. It is a handy little tool and works to perfection without the slightest injury to the plants.

Price, 50c. Postpaid

\section{DAHLIA SEED}

IMPORTANT-It is very important that you understand that every plant you get from seed is an entirely new variety and may be even better than the flower it is taken from. So please do not call any dahlia obtained from seed by any name that is already given to any dahlia as it has no connection with any other.

I do not save seed from poor, insignificant flowers. The wonderful success my customers have with my seed is ample proof that it is far superior to any on the market.

\section{DIRECTIONS FOR PLANTING}

In the latitude of New York City seed should be sown about March 15th to April 1st. Flats or boxes about 3 or 4 inches deep are best. Cover the seed about $1 / 2$ inch deep and keep in a warm place until the plants are all above ground. Then they can be moved to a cooler place. They should be kept in the sun to avoid growing too slender. Transplant in the open when weather is free from frost, about 15 inches apart. Discard any undesirable ones as soon as they bloom, thus giving the others more space.

Be sure your full address comes with every letter or order. 


\section{George L. Stillman's Dahlia \\ PRICE LIST}

PRICES OF SEED IN LARGE PACKAGES AS FOLLOWS:

THE FAMOUS RHODE ISLAND GROUP $\ldots \ldots \ldots \ldots \ldots \ldots \$ 15.00$

THE OCEAN GROUP .................... 10.00

THE NEW YORK GROUP ................... 5.00

SPECIAL MIXTURE NEW SEEDLINGS .......... 5.00

THE TEN SEA LIONS ................... 3.00

THE LOMA FAMILY .................... 3.00

THE INDIAN FAMILY $\ldots \ldots \ldots \ldots \ldots \ldots \ldots \ldots \ldots \ldots .2 .00$

SEEDS FROM ALL WHITE DAHLIAS ........... 2.00

HYBRID CACTUS ..................... 3.00

MIXED DECORATIVE .................... 2.50

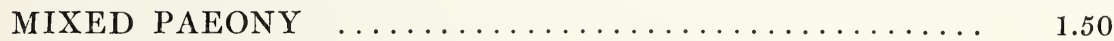

SEED FROM ALL SINGLES $\ldots \ldots \ldots \ldots \ldots \ldots \ldots \ldots \ldots . .50$

GENERAL MIXTURE ..................... 1.00

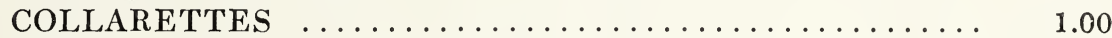

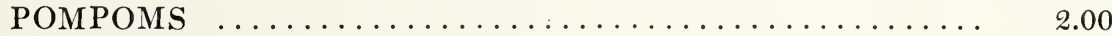

To meet the wants of some of my customers, I have kept the following popular varieties of seed separate. Prices as follows:

Pkg.

THE U. S. A. ......................... $\$ 10.00$

RUTH THOMPSON $\ldots \ldots \ldots \ldots \ldots \ldots \ldots \ldots \ldots \ldots \ldots \ldots, 5.00$

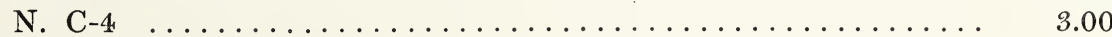

\section{DAHLIA STAKES}

As many of my customers make inquiries of me as to where they may obtain strong Dahlia stakes, I have arranged to furnish a good, strong, Oak Stake, $1 \frac{1}{4}$ inches square, and 6 feet long, in the rough, just as they come from the mill, at 12c. each, F. O. B. Westerly. These stakes will last almost a life time if kept under cover when not in use.

Adclress all communications to

\section{GEORGE L. STILLMAN, Dahlia Specialist} WESTERLY, RHODE ISLAND 


\section{George L. Stilluman's Dahlia PRICE LIST}

\section{GENERAL INSTRUCTIONS}

First of All.- Send me your order as soon as you receive my catalogue, as the demand for some varieties often exceeds the supply, and those who order early are less liable to disappointment than if they waited till the rush at the time of planting. ORDER bulbs sent early to avoid disappointment. Kindly order by name and not by number.

If you do not want to spare all of the money to send with the order, send the order along, together with one-quarter of the amount as a guarantee of good faith, and the balance can be remitted when you receive notice that your order is ready to ship.

Customers who are not personally known to me will be notified when to remit the amount of their bill.

Bulbs are shipped as soon after April 1st as convenient, unless otherwise specified.

When sending orders use the Order Blank furnished with this catalogue. Any number of additional Order Blanks may be had upon application. When filling out the Order Blanks, be sure to fill out the Second Choice columns as well as the First Choice.

It is well to mention the varieties that rou are willing to have substituted in case some of those selected are all sold out. Or, if requested, I will select such varieties as will substitute to best advantage. Otherwise, money sent for such bulbs will be returned to the sender.

Size of Bulbs.-Some rarieties of Dahlias always produce very small bulbs (or tubers), while other rarieties make large ones. So do not consider small bulbs as worthless, for they are equally as good as large ones.

I Guarantee Every Bulb I Sell.-The utmost care is always exercised in selecting every bulb I send out, and every one is believed to have at least one good sprouting eye that will produce a good plant under proper care, or replace it with another.

Bulbs to be replaced after July 1st will be booked for shipment the next season only, and in all cases the bulbs to be replaced must be returned.

I guarantee all bulbs to be true to name and sure to grow at the time they are sint out.

The best way to send money is by registered letter, money order or express order.

Do not hesitate to send small orders. An order for one bulb will be as carefully filled as an order for one thousand.

Terms.-Cash invariably in advance, or C. O. D. On all retail orders fully paid for in advance I will prepay carriage to ans part of the United States. This does not apply to special collections.

ATTENTION.-Orders taken in the Fall not fully paicl for will be sent $C$. O. D., unless previous arrangement has been made.

Do not delay ordering until the time to plant, as many varieties may be sold out before that time. ORDER EARI,Y.

If bulbs are received before it is convenient to plant them, ther should be covered with moist earth until planted.

I use the utmost care to prevent errors in labeling and packing. but if an error does occur. kindly notifv mo, that it mas be rectified immediately.

Please bear in mind that orders are booked and assembled in rotation as received, and are packed to be shipped at the time your order specifies.

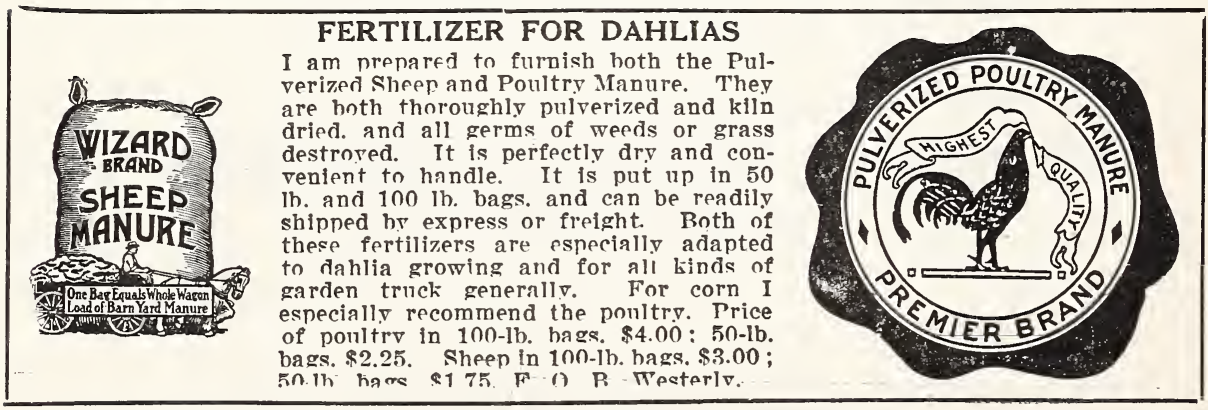





\section{George L. Stilluman's Dahlia PRICE LIST}

\section{POINTERS ON GROWING DAHLIAS}

There are always some people who do not suceced in growing Dahlias with their first season's experience, and are naturally very much disappointed. To those I wish to say that it is not due to a fault of mine or of the bulbs, but is wholly due to your method of cultivation, kind of soil, or weather conditions. A glance through the hundreds of letters I receive from pleased, enthusiastic people who purchase their stock from me is positive proof that my stock is without fault. Exercise a little patience and a whole lot of good common sense and you will grow a: nice Dahlias as anyone.

The Right Kind of Soil.--The kind of soil for Dahlias is a light loam or a stony linoll or side hill.

It is very essential that the soil has good drainage. A rich soil is all right if it has good drainage. Heavy, solid ground will produce an abundance of foliage and few and inferior blossoms.

It should be borne in mind that the poorer the soil, the more fertilizer it requires, and the lighter the soil, the more water it needs. See directions for watering elsewhere. It is a very unwise thing to advise no fertilizer in growing Dahlias, especially in the Eastern states. As a rule nothing will grow without sufficient nourishment. Heavy soil is usually quite rich and when lightened as directed elsewhere, will not need as much fertilizing as weak, light soil.

Heavy Soil.-If your soil is heavy with a clay sub-soil, it should be lightened with coal ashes or sand. If coal ashes are used, the coarser that they are the better, the main object being to make the soil lighter, more porous, and to give it a good drainage. I have seen Dahlias grown to perfection on a spot where the soil was nearly clear coal ashes.

In applying coal ashes they should be plowed, or spaded in, broad cast, 10 to 18 inches deep, and not dumped in one spot where the Dahlia is planted. One part coal ashes, or sand, and one p:irt soil will not lighten it any too much. This will give a better drainage and allow the sun to act on the soil to a greater depth.

Ashes from either hard or soft coal will do but on very heavy soil those from soft coal are preferable, being much coarser. Both kinds mixed together are also desirable.

Watering.-Watering is one of the most essential things in producing perfect blooms on your Dahlias. 90 per cent. of the failures in growing Dahlias is due to lack of water when the hot days of Summer come. Did you ever notice that the stalk of the Dahlia is about all water except the outside shell, and that where sufficient water is supplied the hole in the center of the main stalk is nearly full of water?

The Dahlia, as soon as it begins to bloom, will absorb the moisture from the soil for a distance of two or three feet. For example: Dig down deeply amid the roots of the Dahlia and then again about 4 or 5 feet from the plant and observe the difference. The dry hot days come earlier some years than others, but usually when the buds begin to open it is time to begin watering.

The best method of doing this is to make a basin-shaped mound of earth around the plant, about 2 feet in diameter, and into this basin turn three or four water pails full of water as often as every three or four days. Fertilizer is used to obtain size, water to make richness of color and perfection. Try it.

Planting.-The ground should be plowed or spaded to a depth of 8 to 10 inches. Make the drill to receive the bulb, 12 to 16 inches long and 6 inches deep, and about 6 inches wide. Lay the bulb down flat in the drill and then apply the fertilizer on both sides of the bulb, 4 to 6 inches from the bulb. If pulverized Sheep Manure is used, about one pint to one quart is usually sufficient to each bulb according to the strength of the soil. If poultry manure is used one pint to each plant is sufficient.

It is very desirable to plow in a quantity of stable manure broadcast. Always stick the stake in the ground before covering the bulb and thus avoid piercing any part of the bulb.

Do not hesitate about ordering Dahlia Bulbs on account of distance, for they go safely by mail or express to anv nart of the world. 


\section{George L. Stillinan's Dahlia PRICE LIST}

\section{A TALK FOR THE BEGINNER}

Do not expect to grow as large flowers as an expert the first vear that you try Dahlias, for with any flower you need some experience as well as instruction in order to have great success.

One of $\mathrm{my}$ customers said that he had no luck with Dahlias until he read my catalogue. There he found the very information that he needed, viz.: How to lighten the soil and render it porous.

If sou have a heavy growth of plants and no flowers, it is an indication of too heary soil, or the same result will be encountered on some dry, rich land. In the latter case water is what is needed and in great abundance, say three or four pails of water three times a week to each plant, with some liguid fertilizer once a week.

Notice that after a heary rainfall Dahlias take on a new life and the flowers are fuller in the center and more brilliant in color.

All the Dahlias described in this catalogne as to color and shape are described to the best of $m y$ ability, bu I do not guarantee them to fully meet the ideas of all purchasers in regard to color sbadings.

The best method of planting in the field is to make the distance between. erery other row 3 and 5 feet, respectively, thus affording a passageway on one side of each row after the plant will become large and fill the 3 -foot space. $2 \frac{1}{2}$ to 3 feet apart in the row according to the nature of the plant is about the right distance when 3 - and 5 -foot spaces are used. Do not plant near trees or shrubs. as tree roots will take the strength of the soil from the Dahlia. In the open by themselves is the best place. Aroid the south side of a large building where the sun is extremely hot without a good circulation of air, for the flowers will burn on the back before fully open.

For early flowering they may be planted any time after May 1st or as soon as all danger from frost is passed.

Some people have an idea that the larger the bulb the better the plant it will make. but this is not the case. A small bulb is just as good as, and in many cases better than, a large one.

The Clumps should be divided about the time that rou are to plant in the Spring. At this time 5011 can readily see where the sprouts are and with the use of a knife the head of the clump can be split through between the sprouts, or eyes, taking whatever bulb, or bulbs, would come off with one sprout. One sprout is always better than more. If the sprouts are over 2 inches long when rou plant the bulb it is better to cut them off a half-inch from the bulb and a new sprout will come and make a better one than the slender one that grew in the cellar.

Care of the Plants.-Keep the ground well cultivated and free from weeds. A frequent stirring of the surface is rery beneficial. An application of some standard fertilizer raked into the surface of the soil around the plants two or three times during the season will be very helpful to the development of the flowers.

Never leave more than one or two stalks in a hill. You will have better plants and larger flowers if you will allow only one sprout to grow.

When the buds begin to open a dressing of liquid fertilizer around the plants, several times in a season, will also be beneficial.

Stakes may be used to adrantage to prevent plants from breaking down.

Disbudding. - The proper way to disbud the plants is as follows: If the first. or clomn hud, appears to be weak or defective. cut it off.

Then when flowers appear on branches, cut them for decorative purposes. below one or two joints, thus removing with the flower whatever huds and foliage mav be there. The flowers and foliage cut t?us give a better effert for whatever nurpose ther are to be used. This method is a good invigorator for the nlant and at the same time does all the dishudding required on the majority of Dahlias.

Remember, if bulbs are received before you are read. to plant them, cover them with moist earth to keep them from drving up.

Care of the Bulbs in Winter.-After the frost has killed the foliage, lift the clumps with a spade fork and allow them to dry in the sun about half a day. They should then be stored in a place free from frost. If an old style cellar without cement bottom or heat, they need not be covered, but if ce. mented and heated $b_{\nabla}$ furnace, they should be covered with paper's and cloth to keep the air from them and thus prevent too much shriveling. 